\title{
Phase field approximation of cohesive fracture models
}

\author{
S. Conti ${ }^{\mathrm{a}}$, M. Focardi ${ }^{\mathrm{c}}$, F. Iurlano ${ }^{\mathrm{a}, \mathrm{b}, *}$

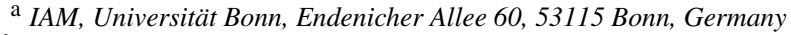 \\ ${ }^{\mathrm{b}}$ HCM, Universität Bonn, Endenicher Allee 60, 53115 Bonn, Germany \\ c DiMaI "U. Dini”, Università degli Studi di Firenze, Viale Morgagni 67/A, 50134 Firenze, Italy
}

Received 27 May 2014; received in revised form 29 January 2015; accepted 27 February 2015

Available online 20 March 2015

\begin{abstract}
We obtain a cohesive fracture model as $\Gamma$-limit, as $\varepsilon \rightarrow 0$, of scalar damage models in which the elastic coefficient is computed from the damage variable $v$ through a function $f_{\varepsilon}$ of the form $f_{\varepsilon}(v)=\min \left\{1, \varepsilon^{\frac{1}{2}} f(v)\right\}$, with $f$ diverging for $v$ close to the value describing undamaged material. The resulting fracture energy can be determined by solving a one-dimensional vectorial optimal profile problem. It is linear in the opening $s$ at small values of $s$ and has a finite limit as $s \rightarrow \infty$. If in addition the function $f$ is allowed to depend on the parameter $\varepsilon$, for specific choices we recover in the limit Dugdale's and Griffith's fracture models, and models with surface energy density having a power-law growth at small openings.
\end{abstract}

๑ 2015 L'Association Publications de l'Institut Henri Poincaré. Published by Elsevier B.V. All rights reserved.

MSC: primary 49J45; secondary 26B30, 74R10, 35A35

Keywords: Cohesive fracture; Phase field models; $\Gamma$-convergence; Damage problems

\section{Introduction}

Variational models in Fracture Mechanics are effectively described through functional spaces with discontinuities. In case of antiplane shear, the corresponding energy functionals take the form

$$
\int_{\Omega} h(|\nabla u|) d x+\int_{J_{u}} g(|[u]|) d \mathcal{H}^{n-1}+\kappa\left|D^{c} u\right|(\Omega)
$$

and the displacement $u: \Omega \rightarrow \mathbb{R}$ is allowed to vary in the space of functions with bounded variation (for more details see $[35,17,11,29])$. The key ingredients in formula (1.1) are respectively a volume term, corresponding to the stored energy and depending on the approximate gradient $\nabla u$, a surface term, modeling the fracture energy and depending on the opening $[u]$ and on the jump set $J_{u}$, and a diffuse term which can be related to micro-cracking and depends on

\footnotetext{
* Corresponding author.

E-mail addresses: sergio.conti@uni-bonn.de (S. Conti), focardi@math.unifi.it (M. Focardi), iurlano@iam.uni-bonn.de (F. Iurlano).
} 
the Cantor derivative $D^{c} u$. In this setting, which is appropriate for the study of monotone deformation processes, the functional (1.1) accounts for both stored energy and the work of dissipation.

Lower semicontinuity is a fundamental property of functionals in the calculus of variations. Problems which are not lower semicontinuous typically lack existence of minimizers and have minimizing sequences which develop fine-scale oscillations or other microstructures. In the case of (1.1), lower semicontinuity requires a relation between the energy densities $h$ and $g$, and the constant $\kappa$. Roughly speaking, $\kappa$ has to agree with the slope of $g$ at 0 and with the slope of $h$ at $+\infty$, see $[4,13]$.

The most renowned example of (1.1) is Griffith's energy, where $h$ is quadratic and $g$ is constant. The semicontinuity condition compels $\kappa$ to be $+\infty$, so that $D^{c} u$ necessarily vanishes and one can take $u$ in the space of special functions with bounded variation. Analytically, the resulting energy coincides with the Mumford-Shah functional for image segmentation. From a physical point of view, the model describes a situation in which already for the smallest opening there is no interaction between the two sides of the crack (brittle fracture [35,17]).

In ductile materials crack proceeds rather through the opening of a series of voids separated by thin filaments, which produce a weak bound between the lips at moderate openings (cohesive fracture $[11,29,33]$ ). The mathematical rephrasing of the corresponding model again involves the functional (1.1), where now $h$ is chosen quadratic near the origin and linear at $+\infty, g$ is concave, linear near the origin, and grows from $g(0)=0$ to some finite value $g(+\infty)$, representing the energetic cost of total fracture. By semicontinuity the constant $\kappa$ is thus finite and non-zero. A particular example is given by Dugdale's energy, where the surface density is precisely $g(s):=\min \{s, 1\}, s \in[0,+\infty)$. Variants of (1.1) which lack lower semicontinuity have been used to study the formation of microcracks [27,28].

A large literature has been devoted to the derivation of models as (1.1) from more regular models, like damage or phase field models, mainly within the framework of $\Gamma$-convergence. These approximations can be interpreted both as microscopic physical models, so that the $\Gamma$-convergence justifies the macroscopic model (1.1), and as regularizations, therefore can be used for example in numerical simulations.

In the first work of this sort, Ambrosio and Tortorelli [9] (see also [8]) showed that the elliptic functional

$$
\int_{\Omega}\left(\left(v^{2}+o(\varepsilon)\right)|\nabla u|^{2}+\frac{(1-v)^{2}}{4 \varepsilon}+\varepsilon|\nabla v|^{2}\right) d x
$$

$\Gamma$-converges in $L^{1}(\Omega) \times L^{1}(\Omega)$ to the Mumford-Shah functional (normalizing the surface coefficient)

$$
\int_{\Omega}|\nabla u|^{2} d x+\mathcal{H}^{n-1}\left(J_{u}\right) .
$$

This result was extended in many directions, for example to vector-valued functions [30,31], to linearized elasticity $[23,24,39]$, to second-order problems [5], to vectorial problems [42], and to models with nonlinear injectivity constraints [37]; for numerical simulations we refer to [12,16,15,21,22]. There is also a large numerical literature on the application to computer vision, see for example [36,10] and references therein. Discrete models for fracture were studied for example in $[19,20]$.

In [2], Alicandro, Braides, and Shah propose an approximation for functionals with more general dependence on the opening of the crack $[u]$, including in particular Barenblatt's cohesive energy (the vector-valued case has been studied in [3]). A key point here is that the regularizations they adopt depend on $|\nabla u|$ through an asymptotically linear function. In particular, their approximating functionals are not lower semicontinuous, and minimizing sequences at fixed $\varepsilon$ are expected to converge to a limit in $B V \times H^{1}$. This limits their usefulness as regularizations of (1.1), since any numerical treatment of the regularized functional would need to treat directly functions with discontinuities, and therefore would not be much simpler than a direct simulation of (1.1). Here we show that (1.1) can be approximated by functionals of the type (1.2), which are quadratic in the gradients and possess a minimizer in the Sobolev space $W^{1,2}$. The quadratic growth of the elastic energy in $\nabla u$ is also classical in damage models, see for example [34,41] and references therein.

The only approximations with quadratic volume energy densities available so far in literature have been obtained for energies which are linear [38] or affine in [u] [7,26,32], and have in common that the profiles of $u$ and $v$ in the optimal-transition problem giving $g(|[u]|)$ can be decoupled.

In this work we obtain a $\Gamma$-convergence result for Barenblatt's cohesive energy with functionals quadratic in $|\nabla u|$. To be precise, we study a damage model similar to those considered in [40,41] (cp. Remark 3.2 below), namely, 


$$
F_{\varepsilon}(u, v):=\int_{\Omega}\left(f_{\varepsilon}^{2}(v)|\nabla u|^{2}+\frac{(1-v)^{2}}{4 \varepsilon}+\varepsilon|\nabla v|^{2}\right) d x,
$$

with $u, v \in H^{1}(\Omega), 0 \leq v \leq 1 \mathcal{L}^{n}$-a.e. in $\Omega$, and $F_{\varepsilon}(u, v):=\infty$ otherwise, and show that it converges to a cohesive fracture model like (1.1), where $g$ is a continuous, subadditive, bounded function with $g(0)=0$, which is linear close to the origin. The potential $f_{\varepsilon}:[0,1) \rightarrow[0,+\infty]$ in $(1.3)$ is defined by

$$
f_{\varepsilon}(z):=1 \wedge \varepsilon^{\frac{1}{2}} f(z),
$$

where $f \in C^{0}([0,1),[0,+\infty))$ is nondecreasing, $f^{-1}(0)=\{0\}$, and it satisfies

$$
\lim _{z \rightarrow 1}(1-z) f(z)=\ell, \quad \ell \in(0,+\infty) .
$$

Our main result describes the asymptotic of $\left(F_{\varepsilon}\right)$ as follows.

Theorem 1.1. Let $\Omega \subset \mathbb{R}^{n}$ be a bounded Lipschitz set, assume (1.3)-(1.5).

Then, the functionals $F_{\varepsilon} \Gamma$-converge in $L^{1}(\Omega) \times L^{1}(\Omega)$ to the functional $F$ defined by

$$
F(u, v):= \begin{cases}\int_{\Omega} h(|\nabla u|) d x+\int_{J_{u}} g(|[u]|) d \mathcal{H}^{n-1}+\ell\left|D^{c} u\right|(\Omega) & \text { if } v=1 \mathcal{L}^{n} \text {-a.e. in } \Omega, u \in G B V(\Omega) \\ +\infty & \text { otherwise } .\end{cases}
$$

Here the volume energy density $h$ is given by $h(s):=s^{2}$ if $s \leq \ell / 2$ and as $h(s):=\ell s-\ell^{2} / 4$ otherwise, while the surface energy density $g$ is given by

$$
\begin{array}{r}
g(s):=\inf \left\{\int_{0}^{1}|1-\beta| \sqrt{f^{2}(\beta)\left|\alpha^{\prime}\right|^{2}+\left|\beta^{\prime}\right|^{2}} d t:(\alpha, \beta) \in H^{1}((0,1)),\right. \\
\alpha(0)=0, \alpha(1)=s, \beta(0)=\beta(1)=1\} .
\end{array}
$$

Let us motivate heuristically the choice of $f_{\varepsilon}$. First note that the truncation by 1 in (1.4) allows to obtain a bulk density in the limit functional which is quadratic near the origin. Indeed, when $f_{\varepsilon}(v)=1$ it is convenient to take $v=1$ and the bulk contribution is exactly $|\nabla u|^{2}$. Instead when $f_{\varepsilon}(v)<1$ and $v$ is close to 1 , it is convenient to optimize the contribution of the first two terms of (1.3) pointwise at given $\nabla u$ (neglecting the contribution of the last term of (1.3)). Supposing that $f(z)=\frac{\ell}{1-z}$, we obtain

$$
\min _{0 \leq z \leq 1}\left\{\varepsilon \frac{\ell^{2}}{(1-z)^{2}}|\nabla u|^{2}+\frac{(1-z)^{2}}{4 \varepsilon}\right\}=\ell|\nabla u| .
$$

The possibility of microstructure (mixtures of the two cases) leads to the convexified bulk density

$$
\left(\min \left\{|\xi|^{2}, \ell|\xi|\right\}\right)^{* *}
$$

that is, $h(|\xi|)$.

The qualitative behavior of the surface density $g$ can be easily related to the choice of $f$. Obviously one easily finds that $g(0)=0$, since the pair $(0,1)$ is optimal. When $s \sim 0$, one still expects that $\beta \sim 1$. In this case we neglect the contribution of the second term in (1.6) and the integral reduces to $s((1-z) f(z))_{z=1}=\ell s$. Therefore, the growth of $f$ near 1 is instrumental to get a linear behavior for $g$ near 0 . When $s \gg 0$ the behavior of $(\alpha, \beta)$ is close to that of an optimal pair for the Ambrosio-Tortorelli approximation (and this is reasonable, since we expect that $g(s)$ tends to a constant as $s \rightarrow+\infty)$. Indeed, in this case one expects that $\left|\alpha^{\prime}\right|$ is large, so that $(1-\beta) f(\beta)$ is compelled to be close to 0 and the first term in (1.6) can be neglected. Hence $\beta$ agrees with a zero of $f$ in the set where $\alpha^{\prime}$ is large (by assumption $f$ is zero just in 0 ) and by the boundary conditions $\beta \sim 1$ near the end points. This gives $g(s) \sim 1$, so that 


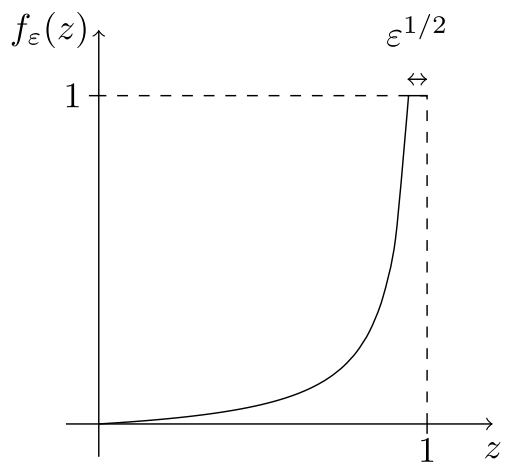

Fig. 1. Sketch of the function $f_{\varepsilon}(z)$ for the prototypical case $f(z)=z /(1-z)$.

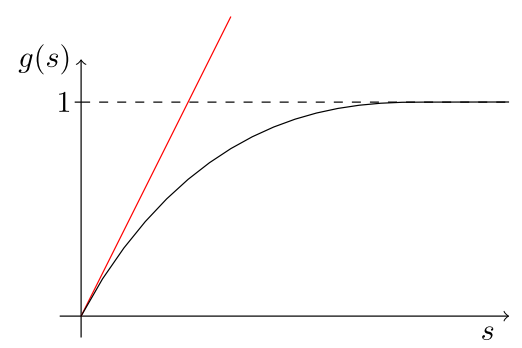

Fig. 2. Sketch of the function $g(s)$ defined in (1.6), obtained by numerical minimization using $f(z)=z /(1-z)$ (cp. Proposition 4.1 and Remark 4.2).

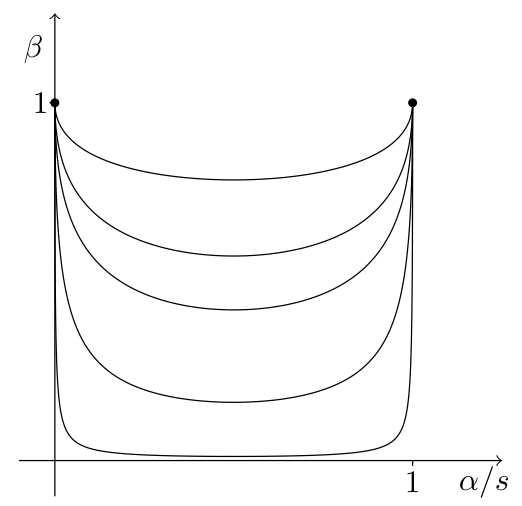

Fig. 3. Optimal profiles $(\alpha / s, \beta)$ obtained numerically from the minimization in the definition of $g(s)$, see $(1.6)$, for $f(z)=z /(1-z)$ and for $s=0.1,0.3,0.5,1$, and 1.5 (from top to bottom). All curves remain inside the square $(0,1) \times(0,1)$ except for the two endpoints.

a sort of interpolation with the function $\ell s$ obtained for small $s$ produces the final $g$. Figs. 1 and 2 show the behavior of $f_{\varepsilon}$ and $g$ in the case $f(z)=z /(1-z)$. Fig. 3 shows the optimal profiles $\alpha, \beta$ in (1.6) as $s$ varies.

One crucial feature of the model we study is that the optimal profiles for the damage variable $v$ and the elastic displacement $u$ cannot be determined separately. They instead arise from a joint vectorial minimization problem which defines the cohesive energy $g$, specified in (1.6). This is analogous to the case analyzed in [2,3].

In closing this Introduction we briefly comment on the methodologies. Theorem 1.1, in the equivalent formulation given in Theorem 3.1 below, is proved first in the one-dimensional case in Section 5, relying on elementary arguments in which we estimate separately the diffuse and jump contributions, and then extended to the general $n$-dimensional setting in Section 6. This extension is obtained by means of several tools. A slicing technique and the above mentioned one-dimensional result are the key for the lower bound inequality. Instead, the upper bound inequality is proved through the direct methods of $\Gamma$-convergence on $S B V$, i.e. abstract compactness results and integral representation of 
the corresponding $\Gamma$-limits. The latter methods are complemented with an ad-hoc one-dimensional construction to match the lower bound on $S B V$ and a relaxation procedure to prove the result on $B V$. Finally, the extension to $G B V$ is obtained via a simple truncation argument.

The issues of equi-coercivity of $F_{\varepsilon}$ and the convergence of the related minima are dealt with in Theorem 3.3 and Corollary 3.4 below, respectively.

Qualitative properties of the surface energy density $g$ defined in (1.6) are analyzed in Section 4. Its monotonicity, sublinearity, boundedness and linear behavior in the origin are established in Proposition 4.1. Proposition 4.3 characterizes $g$ by means of an asymptotic cell formula particularly convenient in the proof of the $\Gamma$-limsup inequality. Furthermore, the dependence of $g$ on $f$ is analyzed in details in Proposition 4.5. The latter results on the one hand show the variety of such a class of functions, and on the other hand are instrumental to handle the limits of sequences in which $f$ depends itself on $\varepsilon$.

In Section 7 we discuss how the phase field approximation scheme can be used to approximate different fracture models. We first consider damage functions of the form

$$
f_{\varepsilon}(z):=\min \left\{1, \varepsilon^{\frac{1}{2}} \max \left\{f(z), a_{\varepsilon} z\right\}\right\}
$$

and show that if $a_{\varepsilon} \rightarrow \infty$ and $a_{\varepsilon} \varepsilon^{\frac{1}{2}} \rightarrow 0$ then a similar result holds with the limiting surface energy $g(s)=1 \wedge(\ell s)$, so that (1.1) reduces to Dugdale's fracture model (Theorem 7.1 in Section 7.1). by

Secondly we consider a situation in which $f$ diverges with exponent $p>1$ close to $z=1$, so that (1.5) is replaced

$$
\lim _{z \rightarrow 1}(1-z)^{p} f(z)=\gamma .
$$

Also in this case the functionals $\Gamma$-converge to a problem of the form (1.1), in this case however the fracture energy $g$ turns out to be proportional to the opening $s$ to the power $2 /(p+1)$ at small $s$. Correspondingly the coefficient $\kappa$ of the diffuse part is infinite, so that the limiting problem is framed in the space GSBV, see Theorem 7.4 in Section 7.2.

Finally we show that if $f_{\varepsilon}(z)$ diverges as $\ell_{\varepsilon} /(1-z)$, with $\ell_{\varepsilon} \rightarrow \infty$, then Griffith's fracture model is recovered in the limit, see Theorem 7.5 in Section 7.3 below.

We finally summarize the structure of the paper. In Section 2 we introduce some notation, some preliminaries, and the functional setting of the problem. The main result of the paper is stated in Section 3, where we also discuss the convergence of related minimum problems and minimizers. Our $\Gamma$-convergence result relies on several properties of the surface energy density $g$ that are established in Section 4. The proof is then given first in the one-dimensional case in Section 5 and then in $n$ dimensions in Section 6. The three generalizations are discussed and proven in Section 7.

\section{Notation and preliminaries}

Let $n \geq 1$ be a fixed integer. We denote the Lebesgue measure and the $k$-dimensional Hausdorff measure in $\mathbb{R}^{n}$ by $\mathcal{L}^{n}$ and $\mathcal{H}^{k}$, respectively. Given $\Omega \subset \mathbb{R}^{n}$ an open bounded set with Lipschitz boundary, we define $\mathcal{A}(\Omega)$ as the set of all open subsets of $\Omega$.

Throughout the paper $c$ denotes a generic positive constant that can vary from line to line.

\section{1. $\Gamma$ - and $\bar{\Gamma}$-convergence}

Given an open set $\Omega \subset \mathbb{R}^{n}$ and a sequence of functionals $\mathscr{F}_{k}: X \times \mathcal{A}(\Omega) \rightarrow[0,+\infty],(X, d)$ a separable metric space, such that the set function $\mathscr{F}_{k}(u ; \cdot)$ is nondecreasing on the family $\mathcal{A}(\Omega)$ of open subsets of $\Omega$, set

$$
\mathscr{F}^{\prime}(x ; A):=\Gamma-\liminf _{k \rightarrow+\infty} \mathscr{F}_{k}(x ; A):=\inf \left\{\liminf _{k \rightarrow+\infty} \mathscr{F}_{k}\left(x_{k} ; A\right): x_{k} \rightarrow z \text { in } X\right\}
$$

and analogously

$$
\mathscr{F}^{\prime \prime}(x ; A):=\Gamma-\limsup _{k \rightarrow+\infty} \mathscr{F}_{k}(x ; A):=\inf \left\{\limsup _{k \rightarrow+\infty} \mathscr{F}_{k}\left(z_{k} ; A\right): x_{k} \rightarrow x \text { in } X\right\}
$$


for every $A \in \mathcal{A}(\Omega)$. We write $\mathscr{F}(x)$ for $\mathscr{F}(x ; \Omega)$, and the same for the other functionals. The sequence $\mathscr{F}_{k}$ $\Gamma$-converges to $\mathscr{F}$ (with respect to the metric $d$ ) if $\mathscr{F}=\mathscr{F}^{\prime}=\mathscr{F}^{\prime \prime}$. The functionals $\mathscr{F}^{\prime}, \mathscr{F}^{\prime \prime}$ are called respectively the $\Gamma$-lower, $\Gamma$-upper limit of the $\mathscr{F}_{k}$ 's.

Next we recall the notion of $\bar{\Gamma}$-convergence, useful in particular to deal with the integral representation of $\Gamma$-limits of families of integral functionals. We say that $\left(\mathscr{F}_{k}\right) \bar{\Gamma}$-converges to $\mathscr{F}: X \times \mathcal{A}(\Omega) \rightarrow[0,+\infty]$ if $\mathscr{F}$ is the inner regular envelope of both functionals $\mathscr{F}^{\prime}$ and $\mathscr{F}^{\prime \prime}$, i.e.,

$$
\mathscr{F}(u ; A)=\sup \left\{\mathscr{F}^{\prime}\left(u ; A^{\prime}\right): A^{\prime} \in \mathcal{A}(\Omega), A^{\prime} \subset \subset A\right\}=\sup \left\{\mathscr{F}^{\prime \prime}\left(u ; A^{\prime}\right): A^{\prime} \in \mathcal{A}(\Omega), A^{\prime} \subset \subset A\right\},
$$

for every $(u, A) \in X \times \mathcal{A}(\Omega)$. These definitions and main results concerning the $\Gamma$-convergence technique can be found in $[25,18]$.

\subsection{Functional setting of the problem}

Our results are set in the spaces $B V$ and $S B V$ and in suitable generalizations. For the definitions, the notation and the main properties of such spaces we refer to the book [6]. We recall that $S B V^{2}(\Omega)$ is defined by

$$
S B V^{2}(\Omega):=\left\{u \in S B V(\Omega): \nabla u \in L^{2}(\Omega) \text { and } \mathcal{H}^{n-1}\left(J_{u}\right)<+\infty\right\} .
$$

A function $u: \Omega \rightarrow \mathbb{R}$ belongs to $G B V(\Omega)$ (respectively to $G S B V(\Omega)$ ) if the truncations $u^{M}:=-M \vee(u \wedge M)$ belong to $B V_{l o c}(\Omega)$ (respectively to $S B V_{l o c}(\Omega)$ ), for every $M>0$. For fine properties of $G B V$ and $G S B V$ again we refer to [6].

The prototype of the asymptotic result we shall prove in Sections 5, 6, and 7 concerns the Mumford-Shah functional of image segmentation

$$
M S(u):= \begin{cases}\int_{\Omega}|\nabla u|^{2} d x+\mathcal{H}^{n-1}\left(J_{u}\right) & \text { if } u \in G S B V(\Omega), \\ +\infty & \text { otherwise in } L^{1}(\Omega) .\end{cases}
$$

Let $\psi:[0,1] \rightarrow[0,1]$ be any nondecreasing lower-semicontinuous function such that $\psi^{-1}(0)=0$ and $\psi(1)=1$. Then the classical approximation by Ambrosio and Tortorelli (cp. [8,9], and also [30]) establishes that the two-field functionals $A T_{k}^{\psi}: L^{1}(\Omega) \times L^{1}(\Omega) \rightarrow[0,+\infty]$

$$
A T_{k}^{\psi}(u, v):= \begin{cases}\int_{\Omega}\left(\psi^{2}(v)|\nabla u|^{2}+\frac{(1-v)^{2}}{4 \varepsilon_{k}}+\varepsilon_{k}|\nabla v|^{2}\right) d x & \text { if }(u, v) \in H^{1}(\Omega) \times H^{1}(\Omega) \\ +\infty & \text { and } 0 \leq v \leq 1 \mathcal{L}^{n} \text {-a.e. in } \Omega, \\ \text { otherwise }\end{cases}
$$

$\Gamma$-converge in $L^{1}(\Omega) \times L^{1}(\Omega)$ to

$$
\widetilde{M S}(u, v):= \begin{cases}M S(u) & \text { if } v=1 \mathcal{L}^{n} \text {-a.e. in } \Omega, \\ +\infty & \text { otherwise, }\end{cases}
$$

that is equivalent to the Mumford-Shah functional $M S$ for minimization purposes.

We finally introduce the notation related to slicing. Fixed $\xi \in \mathbb{S}^{n-1}:=\left\{\xi \in \mathbb{R}^{n}:|\xi|=1\right\}$, let $\Pi^{\xi}:=\left\{y \in \mathbb{R}^{n}\right.$ : $y \cdot \xi=0\}$, and for every subset $A \subset \mathbb{R}^{n}$ set

$$
\begin{aligned}
& A_{y}^{\xi}:=\{t \in \mathbb{R}: y+t \xi \in A\} \quad \text { for } y \in \Pi^{\xi}, \\
& A^{\xi}:=\left\{y \in \Pi^{\xi}: A_{y}^{\xi} \neq \varnothing\right\} .
\end{aligned}
$$

For $u: \Omega \rightarrow \mathbb{R}$ we define the slices $u_{y}^{\xi}: \Omega_{y}^{\xi} \rightarrow \mathbb{R}$ by $u_{y}^{\xi}(t):=u(y+t \xi)$.

Observe that if $u_{k}, u \in L^{1}(\Omega)$ and $u_{k} \rightarrow u$ in $L^{1}(\Omega)$, then for every $\xi \in \mathbb{S}^{n-1}$ there exists a subsequence $\left(u_{k_{j}}\right)$ such that

$$
\left(u_{k_{j}}\right)_{y}^{\xi} \rightarrow u_{y}^{\xi} \text { in } L^{1}\left(\Omega_{y}^{\xi}\right) \text { for } \mathcal{H}^{n-1} \text {-a.e. } y \in \Omega^{\xi} .
$$




\section{The main results: approximation, compactness and convergence of minimizers}

Given a bounded open set $\Omega \subset \mathbb{R}^{n}$ with Lipschitz boundary and an infinitesimal sequence $\varepsilon_{k}>0$, we consider the sequence of functionals $F_{k}: L^{1}(\Omega) \times L^{1}(\Omega) \rightarrow[0,+\infty]$

$$
F_{k}(u, v):= \begin{cases}\int_{\Omega}\left(f_{k}^{2}(v)|\nabla u|^{2}+\frac{(1-v)^{2}}{4 \varepsilon_{k}}+\varepsilon_{k}|\nabla v|^{2}\right) d x & \text { if }(u, v) \in H^{1}(\Omega) \times H^{1}(\Omega) \\ +\infty & \text { and } 0 \leq v \leq 1 \mathcal{L}^{n} \text {-a.e. in } \Omega, \\ \text { otherwise, }\end{cases}
$$

where

$$
f_{k}(z):=1 \wedge \varepsilon_{k}^{\frac{1}{2}} f(z), \quad f_{k}(1)=1,
$$

and

$$
f \in C^{0}([0,1),[0,+\infty)) \text { is a nondecreasing function satisfying } f^{-1}(0)=\{0\}
$$

with

$$
\lim _{z \rightarrow 1^{-}}(1-z) f(z)=\ell, \quad \ell \in(0,+\infty) .
$$

In particular, the function $[0,1) \mapsto(1-z) f(z)$ can be continuously extended to $z=1$ with value $\ell$. One can consider $f(z):=\frac{z}{1-z}$ as prototype.

It is also useful to introduce a localized version $F_{k}(\cdot ; A)$ of $F_{k}$ simply obtained by substituting the domain of integration $\Omega$ with any measurable subset $A$ of $\Omega$ itself. In particular, to be consistent with (3.1), for $A=\Omega$ we shall not indicate the dependence on the domain of integration.

Let now $\Phi: L^{1}(\Omega) \rightarrow[0,+\infty]$ be defined by

$$
\Phi(u):= \begin{cases}\int_{\Omega} h(|\nabla u|) d x+\int_{J_{u}} g(|[u]|) d \mathcal{H}^{n-1}+\ell\left|D^{c} u\right|(\Omega) & \text { if } u \in G B V(\Omega), \\ +\infty & \text { otherwise, }\end{cases}
$$

with $h, g:[0,+\infty) \rightarrow[0,+\infty)$ given by

$$
h(s):= \begin{cases}s^{2} & \text { if } s \leq \ell / 2, \\ \ell s-\ell^{2} / 4 & \text { if } s \geq \ell / 2,\end{cases}
$$

and

$$
g(s):=\inf _{(\alpha, \beta) \in \mathcal{U}_{s}} \int_{0}^{1}|1-\beta| \sqrt{f^{2}(\beta)\left|\alpha^{\prime}\right|^{2}+\left|\beta^{\prime}\right|^{2}} d t,
$$

where $\mathcal{U}_{S}:=\mathcal{U}_{S}(0,1)$ and for all $T>0$

$$
\mathcal{U}_{s}(0, T):=\left\{\alpha, \beta \in H^{1}((0, T)): 0 \leq \beta \leq 1, \alpha(0)=0, \alpha(T)=s, \beta(0)=\beta(T)=1\right\} .
$$

At the points $t$ with $\beta(t)=1$ the integrand in (3.7) reduces to $\ell\left|\alpha^{\prime}\right|(t)$, in agreement with (3.4).

Note that in the one-dimensional setting the functional $\Phi$ turns out to be finite on $B V(\Omega)$ (see Proposition 5.1).

Our main result is the following.

Theorem 3.1. Under the assumptions (3.1)-(3.8), the functionals $F_{k} \Gamma$-converge in $L^{1}(\Omega) \times L^{1}(\Omega)$ to the functional $F$ defined by

$$
F(u, v):= \begin{cases}\Phi(u) & \text { if } v=1 \mathcal{L}^{n} \text {-a.e. in } \Omega \\ +\infty & \text { otherwise }\end{cases}
$$


Remark 3.2. The assumption that $f^{-1}(0)=0$ is not restrictive and changes only the detailed properties of $g$. Indeed, standing all the other assumptions, defining $\lambda:=\sup \{z \in[0,1): f(z)=0\} \in[0,1)$, we would get that $g(s) \leq(1-$ $\lambda)^{2} \wedge \ell s$ (cp. Proposition 4.1 below).

In addition, the potential $(1-z)^{2}$ in $(3.1)$ can be replaced by any continuous, decreasing function $d:[0,1] \rightarrow$ $[0,+\infty)$ with $d(1)=0$. In this case $d^{\frac{1}{2}}(z)$ and $d^{\frac{1}{2}}(\beta)$ appear in formulas (3.4) and (3.7) in place of $1-z$ and $1-\beta$ respectively, and we obtain $g(s) \leq 2 \int_{0}^{1} d^{\frac{1}{2}}(z) d z \wedge \ell s$ (see again Proposition 4.1).

Furthermore, the definition of $f_{k}$ in (3.2) can be given in the following more general form $f_{k}:=\psi_{k} \wedge \varepsilon^{\frac{1}{2}} f$. Here the truncation of $f$ is performed with any continuous nondecreasing function $\psi_{k}:[0,1] \rightarrow[0,1]$ satisfying $\psi_{k} \geq c>0$, and converging uniformly in a neighborhood of $z=1$ to the value 1 .

In conclusion, to highlight the roles of the different terms in the approximation we discuss an explicit example. Consider $f_{k}(z):=\min \left\{\mu, \varepsilon_{k}^{\frac{1}{2}} f(z)\right\}, f$ satisfying (3.3)-(3.4), and $d(z):=v^{2}(1-z)^{2}$, with $\mu$ and $v$ positive constants. According to the discussion above, an elementary scaling argument yields the ensuing energy densities for the $\Gamma$-limit

$$
h_{\mu, \nu}(s):=\left\{\begin{array}{ll}
\mu^{2} s^{2} & \text { if } s \leq v \ell /\left(2 \mu^{2}\right), \\
\nu \ell s-v^{2} \ell^{2} /\left(4 \mu^{2}\right) & \text { if } s>v \ell /\left(2 \mu^{2}\right),
\end{array} \quad g_{\nu}(s):=v g(s) \quad \text { for } s \geq 0 .\right.
$$

We next address the issue of equi-coercivity for the $F_{k}$ 's.

Theorem 3.3. Under the assumptions (3.1)-(3.8), if $\left(u_{k}, v_{k}\right) \in H^{1}(\Omega) \times H^{1}(\Omega)$ is such that

$$
\sup _{k}\left(F_{k}\left(u_{k}, v_{k}\right)+\left\|u_{k}\right\|_{L^{1}(\Omega)}\right)<+\infty,
$$

then there exists a subsequence $\left(u_{j}, v_{j}\right)$ of $\left(u_{k}, v_{k}\right)$ and a function $u \in G B V \cap L^{1}(\Omega)$ such that $u_{j} \rightarrow u \mathcal{L}^{n}$-a.e. in $\Omega$ and $v_{j} \rightarrow 1$ in $L^{1}(\Omega)$.

We shall prove Theorem 3.1 in Sections 5 and 6, Theorem 3.3 shall be established in Section 6.

In the rest of this section instead we address the issue of convergence of minimum problems. Minimum problems related to the functional $F_{k}$ could have no solution due to a lack of coercivity. Therefore we slightly perturb the $f_{k}$ 's to guarantee the existence of a minimum point for each $F_{k}$. This together with Theorems 3.1 and 3.3 shall in turn imply the convergence of minima and minimizers as $k \uparrow \infty$.

Let $\eta_{k}, \varepsilon_{k}$ be positive infinitesimal sequences such that $\eta_{k}=o\left(\varepsilon_{k}\right)$ and let $\zeta \in L^{q}(\Omega)$, with $q>1$. Let us consider the sequence of functionals $G_{k}: L^{1}(\Omega) \times L^{1}(\Omega) \rightarrow[0,+\infty]$ defined by

$$
G_{k}(u, v):=F_{k}(u, v)+\int_{\Omega}\left(\eta_{k}|\nabla u|^{2}+|u-\zeta|^{q}\right) d x
$$

where $F_{k}$ was defined in (3.1). Let now $\mathscr{G}: L^{1}(\Omega) \rightarrow[0,+\infty]$ be defined by

$$
\mathscr{G}(u):=\Phi(u)+\int_{\Omega}|u-\zeta|^{q} d x
$$

where $\Phi$ was defined in (3.5). Then, standard arguments yield the following corollary.

Corollary 3.4. For every $k$, let $\left(u_{k}, v_{k}\right) \in H^{1}(\Omega) \times H^{1}(\Omega)$ be a minimizer of the problem

$$
\min _{(u, v) \in H^{1}(\Omega) \times H^{1}(\Omega)} G_{k}\left(u_{k}, v_{k}\right) .
$$

Then $v_{k} \rightarrow 1$ in $L^{1}(\Omega)$ and a subsequence of $u_{k}$ converges in $L^{q}(\Omega)$ to a minimizer $u$ of the problem

$$
\min _{u \in G B V(\Omega)} \mathscr{G}(u) \text {. }
$$

Moreover the minimum values of (3.10) tend to the minimum value of the limit problem. 


\section{Properties of the surface energy density}

In this section we shall establish several properties of the surface energy density $g$ defined in (3.7).

To this end we shall often exploit that, in computing $g(s), s \geq 0$, we may assume that the admissible functions $\alpha$ satisfy $0 \leq \alpha \leq s$ by a truncation argument (whereas $0 \leq \beta \leq 1$ by definition). Further, the integral appearing in the definition of $g$ is invariant under reparametrizations of $(\alpha, \beta)$.

Proposition 4.1. Under the assumptions (3.2)-(3.4), the function g defined in (3.7)-(3.8) has the following properties:

(i) $g(0)=0$, and $g$ is subadditive, i.e., $g\left(s_{1}+s_{2}\right) \leq g\left(s_{1}\right)+g\left(s_{2}\right)$, for every $s_{1}, s_{2} \in \mathbb{R}^{+}$;

(ii) $g$ is nondecreasing, $0 \leq g(s) \leq 1 \wedge \ell$ f for all $s \in \mathbb{R}^{+}$, and $g$ is Lipschitz continuous with Lipschitz constant $\ell$;

(iii)

$$
\begin{aligned}
& \lim _{s \uparrow \infty} g(s)=1 ; \\
& \lim _{s \downarrow 0} \frac{g(s)}{s}=\ell .
\end{aligned}
$$

Proof. Proof of (i). The pair $(\alpha, \beta)=(0,1)$ is admissible for the minimum problem defining $g(0)$, so that $g(0)=0$.

In order to prove that $g$ is subadditive we fix $s_{1}, s_{2} \in \mathbb{R}^{+}$and we consider the minimum problems for $g\left(s_{1}\right)$ and $g\left(s_{2}\right)$, respectively. Let $\eta>0$ and let $\left(\alpha_{1}, \beta_{1}\right),\left(\alpha_{2}, \beta_{2}\right)$ be admissible pairs respectively for $g\left(s_{1}\right)$ and $g\left(s_{2}\right)$ such that for $i=1,2$

$$
\int_{0}^{1}\left|1-\beta_{i}\right| \sqrt{f^{2}\left(\beta_{i}\right)\left|\alpha_{i}^{\prime}\right|^{2}+\left|\beta_{i}^{\prime}\right|^{2}} d t<g\left(s_{i}\right)+\eta .
$$

Next define $\alpha:=\alpha_{1}$ in $[0,1], \alpha:=\alpha_{2}(\cdot-1)+s_{1}$ in [1,2], $\beta:=\beta_{1}$ in $[0,1]$, and $\beta:=\beta_{2}(\cdot-1)$ in $\left.[1,2]\right)$. An immediate computation and the reparametrization property mentioned above entail the subadditivity of $g$ since $\eta$ is arbitrary.

Proof of (ii). In order to prove that $g$ is nondecreasing we fix $s_{1}, s_{2}$ with $s_{1}<s_{2}$ and $\eta>0$, and we consider $(\alpha, \beta)$ satisfying a condition analogous to (4.3) for $g\left(s_{2}\right)$. Then $\left(\frac{s_{1}}{s_{2}} \alpha, \beta\right)$ is admissible for $g\left(s_{1}\right)$, thus we infer

$$
g\left(s_{1}\right) \leq \int_{0}^{1}|1-\beta| \sqrt{\left(\frac{s_{1}}{s_{2}}\right)^{2} f^{2}(\beta)\left|\alpha^{\prime}\right|^{2}+\left|\beta^{\prime}\right|^{2}} d t<g\left(s_{2}\right)+\eta,
$$

since $s_{1} / s_{2}<1$. As $\eta \rightarrow 0$ we find $g\left(s_{1}\right) \leq g\left(s_{2}\right)$.

Next we prove that $g(s) \leq 1 \wedge \ell$. Indeed, inequality $g \leq 1$ can be derived considering the competitor $(\alpha, \beta)$ given by $\alpha:=0$ in $(0,1 / 3), \alpha:=s$ in $(2 / 3,1)$, and the linear interpolation in $(1 / 3,2 / 3)$, and $\beta:=0$ in $(1 / 3,2 / 3)$ and the linear interpolation to the boundary data 1 in $(0,1 / 3)$ and $(2 / 3,1)$. Moreover, $g(s) \leq \ell s$ for every $s \geq 0$ since the pair $(s t, 1)$ is admissible for $g(s)$.

The Lipschitz continuity of $g$ is an obvious consequence of the facts that $g$ is nondecreasing, subadditive and $g(s) \leq \ell s$ for $s \geq 0$.

Proof of (iii). Let $s_{k}, k \in \mathbb{N}$, be a sequence with $s_{k} \rightarrow \infty$ and let $\left(\alpha_{k}, \beta_{k}\right)$ be an admissible pair for $g\left(s_{k}\right)$ such that

$$
\int_{0}^{1}\left|1-\beta_{k}\right| \sqrt{f^{2}\left(\beta_{k}\right)\left|\alpha_{k}^{\prime}\right|^{2}+\left|\beta_{k}^{\prime}\right|^{2}} d t<g\left(s_{k}\right)+\frac{1}{k} .
$$

If $\inf _{(0,1)} \beta_{k} \geq \delta$ for some $\delta>0$ and for every $k$, then there exists a constant $c(\delta)>0$ such that $f\left(\beta_{k}\right)\left(1-\beta_{k}\right)>c(\delta)$, since $f(z)(1-z) \rightarrow 0$ if and only if $z \rightarrow 0$. Therefore by (4.4) one finds

$$
c(\delta) s_{k} \leq g\left(s_{k}\right)+\frac{1}{k}
$$


so that $g\left(s_{k}\right) \rightarrow+\infty$ as $k \rightarrow+\infty$, this contradicts the fact that $g \leq 1$. Therefore there exists a sequence $x_{k} \in(0,1)$ such that $\liminf _{k} \beta_{k}\left(x_{k}\right)=0$. Since we have already shown that $g \leq 1$, we conclude the proof of (4.1) noticing that (4.4) yields

$$
\left(1-\beta_{k}\left(x_{k}\right)\right)^{2} \leq \int_{0}^{x_{k}}\left|1-\beta_{k}\right|\left|\beta_{k}^{\prime}\right| d t+\int_{x_{k}}^{1}\left|1-\beta_{k}\right|\left|\beta_{k}^{\prime}\right| d t \leq g\left(s_{k}\right)+\frac{1}{k} .
$$

Proof of (iv). Let $s_{k}, k \in \mathbb{N}$, be an infinitesimal sequence and let $\left(\alpha_{k}, \beta_{k}\right)$ be an admissible pair for $g\left(s_{k}\right)$ satisfying (4.4) with $s_{k} / k$ in place of $1 / k$. If there exists $\delta>0$, a not relabeled subsequence of $k$, and a sequence $x_{k} \in[0,1]$ such that $\beta_{k}\left(x_{k}\right)<1-\delta$, then the same computation as in (4.5) leads to

$$
\delta^{2} \leq g\left(s_{k}\right)+\frac{s_{k}}{k} .
$$

As $k \rightarrow+\infty$ this contradicts the fact that $g(s) \leq \ell s$. Therefore, $\beta_{k}$ converges uniformly to 1 and for any $\delta>0$

$$
(\ell-\delta) s_{k} \leq \int_{0}^{1}\left(1-\beta_{k}\right) f\left(\beta_{k}\right)\left|\alpha_{k}^{\prime}\right| d t \leq g\left(s_{k}\right)+\frac{s_{k}}{k}
$$

holds for $k$ sufficiently large, by (3.4). Formula (4.2) immediately follows dividing both sides of the last inequality by $s_{k}$, taking first $k \rightarrow+\infty$ and then $\delta \rightarrow 0$, and using the fact that $g(s) \leq \ell s$, for $s \geq 0$.

Remark 4.2. We can actually show that $g$ does not coincide with the function $1 \wedge \ell s$ at least in the model case $f(z)=\frac{\ell z}{1-z}$ by slightly refining the construction used in (ii) above. With fixed $s>0$, let $\lambda \in[0,1]$ and set $\alpha:=0$ on $[0,1 / 3], \alpha:=s$ on $[1 / 3,2 / 3]$, and the linear interpolation of such values on [1/3,2/3]; moreover, set $\beta_{\lambda}:=\lambda$ on $[1 / 3,2 / 3]$ and the linear interpolation of the values 1 and $\lambda$ on each interval $[0,1 / 3]$ and $[2 / 3,1]$ in order to match the boundary conditions. Straightforward calculations lead to

$$
g(s) \leq(1-\lambda)^{2}+(1-\lambda) f(\lambda) s .
$$

Thus, minimizing over $\lambda \in[0,1]$ yields in turn

$$
g(s) \leq \ell s-\frac{(\ell s)^{2}}{4}<1 \wedge \ell s \quad \text { for all } s \in(0,2 / \ell) .
$$

In what follows it will be convenient to provide an alternative representation of $g$ by means of a cell formula more closely related to the one-dimensional version of the energies $F_{k}$ 's.

To this end we introduce the function $\hat{g}:[0,+\infty) \rightarrow[0,+\infty)$ defined by

$$
\hat{g}(s):=\lim _{T \uparrow \infty} \inf _{(\alpha, \beta) \in \mathcal{U}_{s}(0, T)} \int_{0}^{T}\left(f^{2}(\beta)\left|\alpha^{\prime}\right|^{2}+\frac{|1-\beta|^{2}}{4}+\left|\beta^{\prime}\right|^{2}\right) d t,
$$

where class $\mathcal{U}_{S}(0, T)$ was introduced in (3.8). We note that $\hat{g}$ is well-defined as the minimum problems appearing in its definition are decreasing with respect to $T$.

Proposition 4.3. Under the assumptions (3.2)-(3.4) one has $g=\hat{g}$, where $g$ was defined in (3.7)-(3.8) and $\hat{g}$ in (4.6).

Proof. Let $\alpha, \beta \in H^{1}((0, T)), T>0$, be admissible functions in the definition of $\hat{g}(s)$. By Cauchy's inequality we obtain

$$
|1-\beta| \sqrt{f^{2}(\beta)\left|\alpha^{\prime}\right|^{2}+\left|\beta^{\prime}\right|^{2}} \leq f^{2}(\beta)\left|\alpha^{\prime}\right|^{2}+\left|\beta^{\prime}\right|^{2}+\frac{(1-\beta)^{2}}{4}
$$

and integrating

$$
\int_{0}^{T}|1-\beta| \sqrt{f^{2}(\beta)\left|\alpha^{\prime}\right|^{2}+\left|\beta^{\prime}\right|^{2}} d t \leq \int_{0}^{T}\left(f^{2}(\beta)\left|\alpha^{\prime}\right|^{2}+\frac{|1-\beta|^{2}}{4}+\left|\beta^{\prime}\right|^{2}\right) d t .
$$


The first integral is one-homogeneous in the derivatives, therefore we can reparametrize from $(0, T)$ to $(0,1)$. Taking the infimum over all such $\alpha, \beta$, and $T$ we obtain $g(s) \leq \hat{g}(s)$.

To prove the converse inequality, we first show that $\alpha$ and $\beta$ in the infimum problem defining $g$ can be taken in $W^{1, \infty}((0,1))$. Let $\eta>0$ small and let $\alpha, \beta \in H^{1}((0,1))$ be competitors for $g(s)$ such that

$$
\int_{0}^{1}|1-\beta| \sqrt{f^{2}(\beta)\left|\alpha^{\prime}\right|^{2}+\left|\beta^{\prime}\right|^{2}} d t<g(s)+\eta .
$$

By density we find two sequences $\alpha_{j}, \beta_{j} \in W^{1, \infty}((0,1))$ (actually in $\left.C^{\infty}([0,1])\right)$ such that $\alpha_{j}(0)=0, \alpha_{j}(1)=s$, $\beta_{j}(0)=\beta_{j}(1)=1,0 \leq \beta_{j} \leq 1$, and converging respectively to $\alpha$ and $\beta$ in $H^{1}((0,1))$. Since the function $(1-z) f(z)$ is uniformly continuous and $\beta_{j} \rightarrow \beta$ also uniformly, we deduce that

$$
\int_{0}^{1}\left|1-\beta_{j}\right| \sqrt{f^{2}\left(\beta_{j}\right)\left|\alpha_{j}^{\prime}\right|^{2}+\left|\beta_{j}^{\prime}\right|^{2}} d t<g(s)+\eta
$$

for $j$ large, and this concludes the proof of the claim.

Let us prove now that $\hat{g} \leq g$. We fix a small parameter $\eta>0$ and consider competitors $\alpha, \beta \in W^{1, \infty}((0,1))$ for $g(s)$ satisfying (4.7). We define, for $t \in[0,1]$,

$$
\beta^{\eta}(t):=\beta(t) \wedge(1-\eta) \quad \text { and } \quad \psi_{\eta}(t):=\int_{0}^{t} \frac{2}{1-\beta^{\eta}} \sqrt{\eta+f^{2}\left(\beta^{\eta}\right)\left|\alpha^{\prime}\right|^{2}+\left|\left(\beta^{\eta}\right)^{\prime}\right|^{2}} d t^{\prime} .
$$

The function $\psi_{\eta}:[0,1] \rightarrow\left[0, M_{\eta}:=\psi_{\eta}(1)\right]$ is bilipschitz and in particular invertible. We define $\bar{\alpha}^{\eta}, \bar{\beta}^{\eta} \in$ $W^{1, \infty}\left(\left(0, M_{\eta}\right)\right)$ by

$$
\bar{\alpha}^{\eta}:=\alpha \circ \psi_{\eta}^{-1} \text { and } \bar{\beta}^{\eta}:=\beta^{\eta} \circ \psi_{\eta}^{-1} .
$$

We compute, using the definition and the change of variables $x=\psi_{\eta}(t)$,

$$
\begin{aligned}
\int_{0}^{M_{\eta}} \frac{\left(1-\bar{\beta}^{\eta}\right)^{2}}{4} d x & =\int_{0}^{M_{\eta}} \frac{\left(1-\beta^{\eta}\left(\psi_{\eta}^{-1}(x)\right)\right)^{2}}{4} d x=\int_{0}^{1} \frac{\left(1-\beta^{\eta}(t)\right)^{2}}{4} \psi_{\eta}^{\prime}(t) d t \\
& =\int_{0}^{1} \frac{1-\beta^{\eta}}{2} \sqrt{\eta+f^{2}\left(\beta^{\eta}\right)\left|\alpha^{\prime}\right|^{2}+\left|\left(\beta^{\eta}\right)^{\prime}\right|^{2}} d t \\
& \leq \sqrt{\eta}+\int_{0}^{1} \frac{1-\beta^{\eta}}{2} \sqrt{f^{2}\left(\beta^{\eta}\right)\left|\alpha^{\prime}\right|^{2}+\left|\left(\beta^{\eta}\right)^{\prime}\right|^{2}} d t,
\end{aligned}
$$

where we inserted $\psi_{\eta}^{\prime}$ from the definition of $\psi_{\eta}$ and used $\sqrt{\eta+A} \leq \sqrt{\eta}+\sqrt{A}$. Analogously,

$$
\begin{aligned}
\int_{0}^{M_{\eta}}\left(f^{2}\left(\bar{\beta}^{\eta}\right)\left|\left(\bar{\alpha}^{\eta}\right)^{\prime}\right|^{2}+\left|\left(\bar{\beta}^{\eta}\right)^{\prime}\right|^{2}\right) d x & =\int_{0}^{1}\left(f^{2}\left(\beta^{\eta}\right)\left|\alpha^{\prime}\right|^{2}+\left|\left(\beta^{\eta}\right)^{\prime}\right|^{2}\right) \frac{1}{\psi_{\eta}^{\prime}} d t \\
& =\int_{0}^{1}\left(f^{2}\left(\beta^{\eta}\right)\left|\alpha^{\prime}\right|^{2}+\left|\left(\beta^{\eta}\right)^{\prime}\right|^{2}\right) \frac{1-\beta^{\eta}}{2 \sqrt{\eta+f^{2}\left(\beta^{\eta}\right)\left|\alpha^{\prime}\right|^{2}+\left|\left(\beta^{\eta}\right)^{\prime}\right|^{2}}} d t \\
& \leq \int_{0}^{1} \frac{1-\beta^{\eta}}{2} \sqrt{f^{2}\left(\beta^{\eta}\right)\left|\alpha^{\prime}\right|^{2}+\left|\left(\beta^{\eta}\right)^{\prime}\right|^{2}} d t .
\end{aligned}
$$


We extend $\bar{\alpha}^{\eta}$ and $\bar{\beta}^{\eta}$ to $\left(-1, M_{\eta}+1\right)$ setting $\bar{\alpha}^{\eta}:=0$ in $(-1,0), \bar{\alpha}^{\eta}:=s$ in $\left(M_{\eta}, M_{\eta}+1\right)$, and $\bar{\beta}^{\eta}$ the linear interpolation between $1-\eta$ and 1 in each of the two intervals, so that they obey the required boundary conditions for $\hat{g}$ in the larger interval. Collecting terms, we obtain

$$
\begin{aligned}
\hat{g}(s) & \left.\leq\left.\int_{-1}^{M_{\eta}+1}\left(\frac{\left(1-\bar{\beta}^{\eta}\right)^{2}}{4}+f^{2}\left(\bar{\beta}^{\eta}\right)\left|\bar{\alpha}_{\eta}^{\prime}\right|^{2}+\mid \overline{(} \beta^{\eta}\right)^{\prime}\right|^{2}\right) d x \\
& \leq \sqrt{\eta}+3 \eta^{2}+\int_{0}^{1}\left(1-\beta^{\eta}\right) \sqrt{f^{2}\left(\beta^{\eta}\right)\left|\alpha^{\prime}\right|^{2}+\left|\left(\beta^{\eta}\right)^{\prime}\right|^{2}} d t,
\end{aligned}
$$

where the $3 \eta^{2}$ term comes from an explicit computation on the two boundary intervals.

It remains to replace $\beta^{\eta}$ by $\beta$ in the last integral. We observe that $\left(\beta^{\eta}\right)^{\prime}=0$ almost everywhere on the set where $\beta \neq \beta^{\eta}$ (which coincides with the set $\{\beta>1-\eta\}$ ). Therefore

$$
\begin{aligned}
\int_{\left\{\beta \neq \beta^{\eta}\right\}}\left(1-\beta^{\eta}\right) \sqrt{f^{2}\left(\beta^{\eta}\right)\left|\alpha^{\prime}\right|^{2}+\left|\left(\beta^{\eta}\right)^{\prime}\right|^{2}} d t & =\int_{\left\{\beta \neq \beta^{\eta}\right\}}\left(1-\beta^{\eta}\right) f\left(\beta^{\eta}\right)\left|\alpha^{\prime}\right| d t \\
& \leq \int_{\left\{\beta \neq \beta^{\eta}\right\}}(1-\beta) f(\beta)\left|\alpha^{\prime}\right| d t+\omega(\eta) \int_{0}^{1}\left|\alpha^{\prime}\right| d t
\end{aligned}
$$

where $\omega(\eta)$ is the continuity modulus of $(1-z) f(z)$ near $z=1$, and therefore

$$
\hat{g}(s) \leq \sqrt{\eta}+3 \eta^{2}+\omega(\eta) \int_{0}^{1}\left|\alpha^{\prime}\right| d t+\int_{0}^{1}(1-\beta) \sqrt{f^{2}(\beta)\left|\alpha^{\prime}\right|^{2}+\left|\beta^{\prime}\right|^{2}} d t .
$$

Since the last integral is less than $g(s)+\eta$ and $\eta$ can be made arbitrarily small, this concludes the proof.

For the proof of the lower bound we also need to introduce the auxiliary functions $g^{(\eta)}:[0,+\infty) \rightarrow[0,+\infty)$, for $\eta>0$, defined by

$$
g^{(\eta)}(s):=\inf _{(\alpha, \beta) \in \mathcal{U}_{s}^{(\eta)}} \int_{0}^{1}|1-\beta| \sqrt{f^{2}(\beta)\left|\alpha^{\prime}\right|^{2}+\left|\beta^{\prime}\right|^{2}} d t,
$$

where

$$
\mathcal{U}_{s}^{(\eta)}:=\left\{\alpha, \beta \in H^{1}((0,1)): \alpha(0)=0, \alpha(1)=s, \beta(0)=\beta(1)=1-\eta\right\} .
$$

Proposition 4.4. Under the assumptions (3.2)-(3.4) one has

$$
\left|g(s)-g^{(\eta)}(s)\right| \leq \eta^{2} \text { for all } s \geq 0,
$$

where $g$ was defined in (3.7)-(3.8) and $g^{(\eta)}$ in (4.9).

Proof. We consider the minimum problems for $g$ and $g^{(\eta)}$ respectively in the intervals $(-1,2)$ and $(0,1)$. Let $\left(\alpha_{\eta}, \beta_{\eta}\right)$ be an admissible pair for $g^{(\eta)}(s)$ and let $\alpha:=0$ in $(-1,0), \alpha:=\alpha_{\eta}$ in $(0,1)$, and $\alpha:=s$ in $(1,2)$; we also set $\beta:=\beta_{\eta}$ in $(0,1)$ and linearly linked to 1 in $(-1,0)$ and in $(1,2)$. Then an easy computation shows that

$$
g(s) \leq \int_{0}^{1}\left|1-\beta_{\eta}\right| \sqrt{f^{2}\left(\beta_{\eta}\right)\left|\alpha_{\eta}^{\prime}\right|^{2}+\left|\beta_{\eta}^{\prime}\right|^{2}} d t+\eta^{2} .
$$


By taking the infimum on $\left(\alpha_{\eta}, \beta_{\eta}\right)$ we infer that

$$
g(s) \leq g^{(\eta)}(s)+\eta^{2} .
$$

Reversing the roles of $g$ and $g^{(\eta)}$ we conclude.

Finally, we study the dependence of $g$ on the function $f$ in detail. The results in the next proposition provide a first insight on the class of functions $g$ that arise as surface energy densities in our analysis. Moreover, they will be instrumental to get in the limit different energies by slightly changing the functionals $F_{k}$ 's in (3.1) (cp. Theorems 7.1, 7.4 , and 7.5 below).

Proposition 4.5. Let $\left(f^{(j)}\right)$ be a sequence of functions satisfying (3.3) and (3.4). Denote by $\ell_{j}, g_{j}$ the value of the limit in (3.4) and the function in (3.7) corresponding to $f^{(j)}$, respectively. Then,

(i) if $\ell_{j}=\ell$ for all $j, f^{(j)} \geq f^{(j+1)}$, and $f^{(j)}(z) \downarrow 0$ for all $z \in[0,1)$, then $g_{j} \geq g_{j+1}$ and $g_{j}(s) \downarrow 0$ for all $s \in[0,+\infty)$;

(ii) if $\ell_{j}=\ell$ for all $j, f^{(j)} \leq f^{(j+1)}$, and $f^{(j)}(z) \uparrow \infty$ for all $z \in(0,1)$, then $g_{j} \leq g_{j+1}$ and $g_{j}(s) \uparrow 1 \wedge \ell$ for all $s \in[0,+\infty)$

(iii) if $\ell_{j} \uparrow \infty, f^{(j)} \leq f^{(j+1)}$, and $f^{(j)}(z) \uparrow \infty$ for all $z \in(0,1)$, then $g_{j} \leq g_{j+1}$ and $g_{j}(s) \rightarrow \chi_{(0,+\infty)}(s)$ for all $s \in[0,+\infty)$.

Proof. To prove item (i) we note that the monotonicity of the sequence $\left(f^{(j)}\right)$ and the pointwise convergence to a continuous function on $[0,1)$ yield that the sequence $\left(f^{(j)}\right)$ actually converges uniformly on compact subsets of $[0,1)$ to 0 . Therefore, for all $\delta \in(0,1)$ we have for some $j_{\delta}$

$$
\max _{[0,1-\delta]} f^{(j)} \leq \delta \quad \text { for all } j \geq j_{\delta}
$$

Then, consider $\alpha_{j}, \beta_{j}$ defined as follows: $\alpha_{j}(t):=3 s(t-1 / 3)$ on $[1 / 3,2 / 3], \alpha_{j}:=0$ on $[0,1 / 3]$, and $\alpha_{j}:=s$ on $[2 / 3,1] ; \beta_{j}:=1-\delta$ on $[1 / 3,2 / 3]$ and a linear interpolation between the values 1 and $1-\delta$ on each interval $[0,1 / 3]$ and $[2 / 3,1]$. Straightforward calculations give

$$
g_{j}(s) \leq \delta^{2} s+\delta^{2} \quad \text { for all } j \geq j_{\delta},
$$

from which the conclusion follows by passing to the limit first in $j \uparrow+\infty$ and finally letting $\delta \downarrow 0$.

We now turn to item (ii). We first note that the sequence $\left(g_{j}\right)$ is nondecreasing and that

$$
\lim _{j} g_{j}(s) \leq \ell s \wedge 1
$$

in view of item (ii) in Proposition 4.1. Next we show the following: for all $\delta>0$

$$
\lim _{j} \min _{z \in[\delta, 1]}(1-z) f^{(j)}(z)=\ell .
$$

Let $z_{j} \in \operatorname{argmin}_{[\delta, 1]}(1-z) f^{(j)}(z)$, and denote by $j_{k}$ a subsequence such that

$$
\lim _{k} \min _{z \in[\delta, 1]}(1-z) f^{\left(j_{k}\right)}(z)=\liminf \min _{z \in[\delta, 1]}(1-z) f^{(j)}(z) .
$$

Either $\lim \sup _{k} z_{j_{k}}<1$ or $\lim \sup _{k} z_{j_{k}}=1$. We exclude the former possibility: suppose that, up to further subsequences not relabeled, $\lim _{k} z_{j_{k}}=z_{\infty} \in[\delta, 1)$, then for all $i \in \mathbb{N}$

$$
\liminf _{k}\left(1-z_{j_{k}}\right) f^{\left(j_{k}\right)}\left(z_{j_{k}}\right)=\left(1-z_{\infty}\right) \liminf _{k} f^{\left(j_{k}\right)}\left(z_{j_{k}}\right) \geq\left(1-z_{\infty}\right) f^{(i)}\left(z_{\infty}\right),
$$

that gives a contradiction by letting $i \uparrow \infty$ since by minimality of $z_{j}$

$$
\left(1-z_{j}\right) f^{(j)}\left(z_{j}\right) \leq \ell \quad \text { for all } j .
$$


Therefore, $\lim \sup _{k} z_{j_{k}}=1$, and thus we get

$$
\liminf _{j}\left(1-z_{j}\right) f^{(j)}\left(z_{j}\right) \geq \liminf _{k}\left(1-z_{j_{k}}\right) f^{(1)}\left(z_{j_{k}}\right)=\ell .
$$

Formula (4.11) follows straightforwardly by this and (4.12).

Clearly $g_{j}(0)=0$ for all $j$. Let then $s \in(0,+\infty)$ and $\left(\alpha_{j}, \beta_{j}\right) \in \mathcal{U}_{s}$ be such that

$$
g_{j}(s)+\frac{1}{j} \geq \int_{0}^{1}\left|1-\beta_{j}\right| \sqrt{\left(f^{(j)}\right)^{2}\left(\beta_{j}\right)\left|\alpha_{j}^{\prime}\right|^{2}+\left|\beta_{j}^{\prime}\right|^{2}} d t .
$$

There are now two possibilities: either there exists $\delta>0$ and a subsequence $j_{k}$ such that inf $[0,1] \beta_{j_{k}} \geq \delta$, or $\inf _{[0,1]} \beta_{j} \rightarrow 0$. In the former case the subsequence satisfies

$$
g_{j_{k}}(s)+\frac{1}{j_{k}} \geq\left(\min _{z \in[\delta, 1]}(1-z) f^{\left(j_{k}\right)}(z)\right) s .
$$

Taking the $\limsup _{k}$ and using (4.11) we obtain

$$
\limsup _{j} g_{j}(s) \geq \ell s .
$$

In the other case for every $\delta>0$ definitively it holds

$$
g_{j}(s)+\frac{1}{j} \geq \int_{0}^{1}\left(1-\beta_{j}\right)\left|\beta_{j}^{\prime}\right| d t \geq(1-\delta)^{2} .
$$

Taking again the lim sup we obtain

$$
\limsup _{j} g_{j}(s) \geq(1-\delta)^{2} .
$$

Since $\delta$ was arbitrary, from (4.13) and (4.15) we obtain $\limsup _{j} g_{j}(s) \geq 1 \wedge \ell s$ and, recalling (4.10) and $g_{j} \leq g_{j+1}$, conclude the proof of (ii).

Let us now prove item (iii). First we observe that $g_{j}(s) \leq 1$ for all $j$. To prove the lower bound, we notice that arguing similarly as in the proof of (4.11) one obtains

$$
\lim _{j} \min _{z \in[\delta, 1]}(1-z) f^{(j)}(z)=+\infty \text { for all } \delta>0 .
$$

For any $s \in(0,+\infty)$ we choose $\left(\alpha_{j}, \beta_{j}\right) \in \mathcal{U}_{s}$ such that

$$
g_{j}(s)+\frac{1}{j} \geq \int_{0}^{1}\left|1-\beta_{j}\right| \sqrt{\left(f^{(j)}\right)^{2}\left(\beta_{j}\right)\left|\alpha_{j}^{\prime}\right|^{2}+\left|\beta_{j}^{\prime}\right|^{2}} d t .
$$

If there is $\delta>0$ such that inf $\beta_{j} \geq \delta$ for infinitely many $j$ then for the same indices

$$
g_{j}(s)+\frac{1}{j} \geq \min _{z \in[\delta, 1]}(1-z) f^{(j)}(z) s,
$$

which in view of (4.16) and the bound $g_{j}(s) \leq 1$ is impossible. Therefore $\inf _{[0,1]} \beta_{j} \rightarrow 0$, which in view of (4.14) proves the assertion.

Remark 4.6. The monotonicity assumption $f^{(j)} \leq f^{(j+1)}$ in items (ii) and (iii) above leads to simple proofs but it is actually not needed. The same convergence results for $\left(g_{j}\right)$ would follow by using the uniform convergence on compact subsets of $[0,1)$ of $\left(f^{(j)}\right)$. The latter property is a consequence of the fact that each $f^{(j)}$ is nondecreasing and that $f \in C^{0}([0,1))$. 


\section{Proof in the one-dimensional case}

Let us study first the one-dimensional case $n=1$. As usual, we will prove a $\Gamma$-liminf inequality and a $\Gamma$-limsup inequality. The following proposition gives the lower estimate.

Proposition 5.1 (Lower bound). Under the assumptions (3.1)-(3.8), for every $(u, v) \in L^{1}(\Omega) \times L^{1}(\Omega)$ it holds

$$
F(u, v) \leq F^{\prime}(u, v),
$$

where $F^{\prime}$ denotes the $\Gamma$-liminf of the sequence $F_{k}$ and $F$ the functional defined in (3.9).

Proof. The conclusion is equivalent to the following fact: let $\left(u_{k}, v_{k}\right)$ be a sequence such that

$$
\begin{aligned}
& \left(u_{k}, v_{k}\right) \rightarrow(u, v) \text { in } L^{1}(\Omega) \times L^{1}(\Omega), \\
& \sup _{k} F_{k}\left(u_{k}, v_{k}\right)<+\infty,
\end{aligned}
$$

then $u \in B V(\Omega), v=1 \mathcal{L}^{1}$-a.e. in $\Omega$, and

$$
\Phi(u) \leq \liminf _{k \rightarrow \infty} F_{k}\left(u_{k}, v_{k}\right)
$$

Since the left-hand side of (5.3) is $\sigma$-additive and the right-hand side is $\sigma$-superadditive with respect to $\Omega$, it is enough to prove the result when $\Omega$ is an interval. For the sake of convenience in what follows we assume $\Omega=(0,1)$.

By (5.2) one deduces that $v=1 \mathcal{L}^{n}$-a.e. in $\Omega$. Up to subsequences one can assume that the lower limit in (5.3) is in fact a limit and that the convergences in (5.1) are also $\mathcal{L}^{1}$-a.e. in $\Omega$.

For the first part of the proof we will use a discretization argument, following the lines of [2]. We fix $\delta \in(0,1)$ and for any $N \in \mathbb{N}$ divide $\Omega$ into $N$ intervals

$$
I_{N}^{j}:=\left(\frac{j-1}{N}, \frac{j}{N}\right), \quad j=1, \ldots, N .
$$

Up to subsequences we can assume that $\lim _{k \rightarrow+\infty} \inf _{I_{N}^{j}} v_{k}$ exists for every $j=1, \ldots, N$. We define

$$
J_{N}:=\left\{j \in\{1, \ldots, N\}: \lim _{k \rightarrow+\infty} \inf _{I_{N}^{j}} v_{k} \leq 1-\delta\right\} .
$$

For a given $j \in J_{N}$, we denote by $x_{k}$ and $y$ two points in $I_{N}^{j}$ such that $v_{k}\left(x_{k}\right)<1-\delta / 2$ and $v_{k}(y) \rightarrow 1$. Then by Cauchy's inequality we deduce for $k$ large (assuming for instance $x_{k} \leq y$ )

$$
\int_{x_{k}}^{y}\left(\frac{\left(1-v_{k}\right)^{2}}{4 \varepsilon_{k}}+\varepsilon_{k}\left|v_{k}^{\prime}\right|^{2}\right) d x \geq \frac{1}{2}\left(\left(1-v_{k}\left(x_{k}\right)\right)^{2}-\left(1-v_{k}(y)\right)^{2}\right) \geq \frac{\delta^{2}}{16} .
$$

The previous computation entails

$$
\sup _{N} \mathcal{H}^{0}\left(J_{N}\right)<+\infty
$$

so that up to subsequences we can assume $J_{N}=\left\{j_{1}^{N}, \ldots, j_{L}^{N}\right\}$, with $L$ independent of $N$, and that all sequences $j_{i}^{N} / N$ converge. We denote by $S$ the set of limits of these sequences,

$$
S=\left\{t_{1}, \ldots, t_{L^{\prime}}\right\}=\left\{\lim _{N \rightarrow+\infty} \frac{j_{i}^{N}}{N}, i=1, \ldots, L\right\} \subset \Omega .
$$

We claim now that there exists a modulus of continuity $\omega$, i.e., $\omega(\delta) \rightarrow 0$ as $\delta \rightarrow 0$, depending only on $f$, such that for all $\eta$ sufficiently small (depending on $\delta$ ) and $k$ sufficiently large (depending on $\eta$ ) one has

$$
(1-\omega(\delta)) \int_{\Omega \backslash S_{\eta}} h\left(\left|u_{k}^{\prime}\right|\right) d x \leq F_{k}\left(u_{k}, v_{k}, \Omega \backslash S_{\eta}\right),
$$


where $S_{\eta}:=\bigcup_{i=1}^{L^{\prime}}\left(t_{i}-\eta, t_{i}+\eta\right)$. It suffices to prove (5.5) in the case that $\eta$ is so small that the intervals $\left(t_{i}-\eta, t_{i}+\eta\right)$ are pairwise disjoint subsets of $\Omega$.

In order to prove (5.5), we observe that by definition of $f_{k}$ in (3.2) and by Cauchy's inequality we obtain

$$
\begin{aligned}
F_{k}\left(u_{k}, v_{k} ; \Omega \backslash S_{\eta}\right) & \geq \int_{\Omega \backslash S_{\eta}}\left(f_{k}^{2}\left(v_{k}\right)\left|u_{k}^{\prime}\right|^{2}+\frac{\left(1-v_{k}\right)^{2}}{4 \varepsilon_{k}}\right) d x \\
& \geq \int_{\Omega \backslash S_{\eta}}\left(\left|u_{k}^{\prime}\right|^{2} \wedge\left(\varepsilon_{k} f^{2}\left(v_{k}\right)\left|u_{k}^{\prime}\right|^{2}+\frac{\left(1-v_{k}\right)^{2}}{4 \varepsilon_{k}}\right)\right) d x \\
& \geq \int_{\Omega \backslash S_{\eta}}\left|u_{k}^{\prime}\right|^{2} \wedge\left(\left(1-v_{k}\right) f\left(v_{k}\right)\left|u_{k}^{\prime}\right|\right) d x .
\end{aligned}
$$

Let us note that $v_{k}>1-\delta$ in $\Omega \backslash S_{\eta}$ for $k$ large. By (3.4) there exists a modulus of continuity $\omega$ such that

$$
|(1-z) f(z)-\ell| \leq \ell \omega(\delta), \quad \text { for } z \geq 1-\delta .
$$

Therefore by (5.6) and (5.7) we obtain

$$
F_{k}\left(u_{k}, v_{k} ; \Omega \backslash S_{\eta}\right) \geq(1-\omega(\delta)) \int_{\Omega \backslash S_{\eta}}\left|u_{k}^{\prime}\right|^{2} \wedge \ell\left|u_{k}^{\prime}\right| d x \geq(1-\omega(\delta)) \int_{\Omega \backslash S_{\eta}} h\left(\left|u_{k}^{\prime}\right|\right) d x .
$$

The last inequality holds true as $h$ is the convex envelope of $t \mapsto t^{2} \wedge \ell t$. Formula (5.8) proves the claim in (5.5).

Notice that the boundedness assumption in (5.2) and formula (5.5) imply that

$$
\sup _{\eta} \sup _{k} \int_{\Omega \backslash S_{\eta}}\left|u_{k}^{\prime}\right| d x<+\infty .
$$

Therefore $u \in B V\left(\Omega \backslash S_{\eta}\right)$, and actually the finiteness of $S$ ensures that $u \in B V(\Omega)$. In addition, the $L^{1}$-lower semicontinuity of the functional $\Phi$ defined in (3.5) yields

$$
(1-\omega(\delta)) \Phi\left(u ; \Omega \backslash S_{\eta}\right) \leq \liminf _{k} F_{k}\left(u_{k}, v_{k} ; \Omega \backslash S_{\eta}\right) .
$$

We now estimate the energy contribution on $S_{\eta}$. To this end it is not restrictive to assume that $S \subseteq J_{u}$.

Let us fix $i \in\left\{1, \ldots, L^{\prime}\right\}$ and consider $I_{\eta}^{i}:=\left(t_{i}-\eta, t_{i}+\eta\right)$. We claim that

$$
(1-\omega(\delta)) g\left(\underset{I_{\eta}^{i}}{\operatorname{ess} \sup } u-\underset{I_{\eta}^{i}}{\operatorname{essinf}} u\right) \leq \liminf _{k \rightarrow+\infty} F_{k}\left(u_{k}, v_{k} ; I_{\eta}^{i}\right)+O(\eta) .
$$

Let us introduce a small parameter $\mu>0$ and $x_{1}, x_{2} \in I_{\eta}^{i}$ such that

$$
\begin{aligned}
v_{k}\left(x_{1}\right) \rightarrow 1, & v_{k}\left(x_{2}\right) \rightarrow 1, \\
u_{k}\left(x_{1}\right) \rightarrow u\left(x_{1}\right), & u_{k}\left(x_{2}\right) \rightarrow u\left(x_{2}\right), \\
u\left(x_{1}\right)>\underset{I_{\eta}^{i}}{\operatorname{ess} \sup u-\mu,} & u\left(x_{2}\right)<\underset{I_{\eta}^{i}}{\operatorname{essinf}} u+\mu .
\end{aligned}
$$

Assuming without loss of generality that $x_{1}<x_{2}$, we define $I:=\left(x_{1}, x_{2}\right)$.

There are just finitely many connected components of the set

$$
\left\{x \in I: v_{k}(x)<1-\eta\right\}
$$

where $v_{k}$ achieves the value $1-\delta$, as a computation analogous to (5.4) easily shows (recall that $\eta \ll \delta$ ). Precisely one finds up to subsequences that the number $N$ of these components is

$$
N \leq \frac{c}{\delta^{2}-\eta^{2}}
$$


for some constant $c>0$ independent of $k$. Let us now estimate the functional $F_{k}$ over each component $C_{k}^{j}$ of this type, $j=1, \ldots, N$. Since $v_{k}<1-\eta$ in $C_{k}^{j}$ one finds for $k$ large that $f_{k}\left(v_{k}\right)=\varepsilon_{k}^{\frac{1}{2}} f\left(v_{k}\right)$, so that for $j=1, \ldots, N$ it follows

$$
\begin{aligned}
F_{k}\left(u_{k}, v_{k} ; C_{k}^{j}\right) & \geq \int_{C_{k}^{j}}\left(\varepsilon_{k} f^{2}\left(v_{k}\right)\left|u_{k}^{\prime}\right|^{2}+\frac{\left(1-v_{k}\right)^{2}}{4 \varepsilon_{k}}+\varepsilon_{k}\left|v_{k}^{\prime}\right|^{2}\right) d x \\
& \geq g^{(\eta)}\left(\left|\int_{C_{k}^{j}} u_{k}^{\prime} d x\right|\right) \geq g\left(\left|\int_{C_{k}^{j}} u_{k}^{\prime} d x\right|\right)-\eta^{2},
\end{aligned}
$$

by Cauchy's inequality and Proposition 4.4.

Outside the selected components $C_{k}^{j}, j=1, \ldots, N$, one has $v_{k} \geq 1-\delta$, so that estimate (5.8) holds with $I \backslash \bigcup_{j=1}^{N} C_{k}^{j}$ replacing $\Omega \backslash S_{\eta}$. Therefore

$$
\begin{aligned}
F_{k}\left(u_{k}, v_{k} ; I \backslash \bigcup_{j=1}^{N} C_{k}^{j}\right) & \geq(1-\omega(\delta)) \int_{I \backslash \bigcup_{j=1}^{N} C_{k}^{j}} h\left(\left|u_{k}^{\prime}\right|\right) d x \\
& \geq(1-\omega(\delta)) \ell \int_{I \backslash \bigcup_{j=1}^{N} C_{k}^{j}}\left|u_{k}^{\prime}\right| d x-(1-\omega(\delta)) \frac{\ell^{2}}{4} \mathcal{L}^{1}\left(I \backslash \bigcup_{j=1}^{N} C_{k}^{j}\right) \\
& \geq(1-\omega(\delta)) g\left(\left|\int_{I \backslash \bigcup_{j=1}^{N} C_{k}^{j}} u_{k}^{\prime} d x\right|\right)-\frac{\ell^{2}}{2} \eta,
\end{aligned}
$$

where we have used the definition of $h$ in (3.6) and Proposition 4.1(ii).

By (5.13), (5.14), and the subadditivity of $g$ one finds

$$
F_{k}\left(u_{k}, v_{k} ; I\right)+\frac{\ell^{2}}{2} \eta+\frac{c \eta^{2}}{\delta^{2}-\eta^{2}} \geq(1-\omega(\delta)) g\left(\left|\int_{I} u_{k}^{\prime} d x\right|\right)=(1-\omega(\delta)) g\left(\left|u_{k}\left(x_{1}\right)-u_{k}\left(x_{2}\right)\right|\right) .
$$

By property (5.11) and by the continuity of $g$, as $k \rightarrow+\infty$ one deduces

$$
\liminf _{k \rightarrow+\infty} F_{k}\left(u_{k}, v_{k} ; I_{\eta}^{i}\right)+\frac{\ell^{2}}{2} \eta+\frac{c \eta^{2}}{\delta^{2}-\eta^{2}} \geq(1-\omega(\delta)) g\left(\left|u\left(x_{1}\right)-u\left(x_{2}\right)\right|\right) .
$$

Finally property (5.12) concludes the proof of (5.10) as $\mu \rightarrow 0$.

Summing (5.9) and (5.10) for $i=1, \ldots, L$ and taking first $\eta \rightarrow 0$ and finally $\delta \rightarrow 0$ concludes the proof.

Proposition 5.2 (Upper bound). Under the assumptions (3.1)-(3.8), for every $(u, v) \in L^{1}(\Omega) \times L^{1}(\Omega)$ it holds

$$
F^{\prime \prime}(u, v) \leq F(u, v),
$$

where $F^{\prime \prime}$ is the $\Gamma$-limsup of the sequence $F_{k}$ and $F$ the functional defined in (3.9).

Proof. Let us consider first the case when $u \in S B V^{2}(\Omega)$. By a localization argument it is not restrictive to assume that $J_{u}=\left\{x_{0}\right\}$ and to take $x_{0}=0$. We also assume for a while that $u$ only takes the two values $u^{ \pm}(0)$ in a neighborhood of 0 .

With fixed $\eta>0$, we consider $T_{\eta}>0$ and $\alpha_{\eta}, \beta_{\eta} \in H^{1}\left(\left(0, T_{\eta}\right)\right)$ such that $\alpha_{\eta}(0)=u^{-}(0), \alpha_{\eta}\left(T_{\eta}\right)=u^{+}(0), 0 \leq$ $\beta_{\eta} \leq 1, \beta_{\eta}(0)=\beta_{\eta}\left(T_{\eta}\right)=1$, and 


$$
g(|[u](0)|)+\eta>\int_{0}^{T_{\eta}}\left(f^{2}\left(\beta_{\eta}\right)\left|\alpha_{\eta}^{\prime}\right|^{2}+\frac{\left|1-\beta_{\eta}\right|^{2}}{4}+\left|\beta_{\eta}^{\prime}\right|^{2}\right) d t .
$$

This choice is possible in view of Proposition 4.3, up to a translation of the variable $\alpha_{\eta}$.

Let us define $A_{k}:=\left(-\frac{\varepsilon_{k} T_{\eta}}{2}, \frac{\varepsilon_{k} T_{\eta}}{2}\right)$ and

$$
\begin{aligned}
& u_{k}(x):= \begin{cases}\alpha_{\eta}\left(\frac{x}{\varepsilon_{k}}+\frac{T_{\eta}}{2}\right) & \text { if } x \in A_{k}, \\
u & \text { otherwise, }\end{cases} \\
& v_{k}(x):= \begin{cases}\beta_{\eta}\left(\frac{x}{\varepsilon_{k}}+\frac{T_{\eta}}{2}\right) & \text { if } x \in A_{k}, \\
1 & \text { otherwise. }\end{cases}
\end{aligned}
$$

An easy computation shows that $\left(u_{k}, v_{k}\right) \rightarrow(u, 1)$ in $L^{1}(\Omega) \times L^{1}(\Omega)$, that $u_{k}, v_{k} \in H^{1}(\Omega)$ for $k$ large, and that for the same $k$

$$
F_{k}\left(u_{k}, v_{k}, \Omega \backslash A_{k}\right) \leq \int_{\Omega}\left|u^{\prime}\right|^{2} d x,
$$

being $f_{k} \leq 1$. Moreover using that $f_{k} \leq \varepsilon_{k}^{\frac{1}{2}} f$ and changing the variable $x$ with $y=\frac{x}{\varepsilon_{k}}+\frac{T_{\eta}}{2}$ one has

$$
F_{k}\left(u_{k}, v_{k}, A_{k}\right) \leq g(|[u](0)|)+\eta,
$$

where we have used (5.15). Therefore we find

$$
F^{\prime \prime}(u, 1) \leq \int_{\Omega}\left|u^{\prime}\right|^{2} d x+\int_{J_{u}}(g(|[u]|)+\eta) d \mathcal{H}^{0},
$$

where $F^{\prime \prime}$ denotes the $\Gamma$-limsup of $F_{k}$ according to Section 2.1, and then

$$
F^{\prime \prime}(u, 1) \leq \Phi(u),
$$

since $\eta$ is arbitrary.

Let us remove now the hypothesis that $u$ is constant near 0 . For a function $u \in S B V^{2}(\Omega)$ with $J_{u}=\{0\}$, one can consider the sequence $u_{j}:=u$ in $\Omega \backslash(-1 / j, 1 / j)$, with $u_{j}:=u(-1 / j)$ in $(-1 / j, 0)$ and $u_{j}=u(1 / j)$ in $(0,1 / j)$. Then $u_{j} \rightarrow u$ in $L^{1}(\Omega)$ and $\left|u_{j}^{\prime}\right| \leq\left|u^{\prime}\right| \mathcal{L}^{1}$-a.e. in $\Omega$, so that by the lower semicontinuity of $F^{\prime \prime}$ and by the absolute continuity of $u$ on both sides of 0 we conclude as $j \rightarrow+\infty$ that $u$ still satisfies (5.16).

The extension of (5.16) to each $u \in S B V^{2}(\Omega)$ with $\mathcal{H}^{0}\left(J_{u}\right)<+\infty$ is immediate and finally [13, Propositions 3.3-3.5] conclude the proof.

\section{Proof in the $n$-dimensional case}

In this section we establish the $\Gamma$-convergence result in the $n$-dimensional setting.

We recover the lower bound estimate by using a slicing technique thus reducing ourselves to the one-dimensional setting of Proposition 5.1. Instead, the upper bound inequality follows by an abstract approach based on integral representation results (cp. Proposition 6.4 below).

Proposition 6.1. Under the assumptions (3.1)-(3.8), for every $(u, v) \in L^{1}(\Omega) \times L^{1}(\Omega)$ it holds

$$
F(u, v) \leq F^{\prime}(u, v)
$$

where $F^{\prime}$ denotes the $\Gamma$-liminf of the sequence $F_{k}$ and $F$ the functional defined in (3.9).

Proof. Let us assume first that $u \in L^{\infty}(\Omega)$. We set $M:=\|u\|_{L^{\infty}(\Omega)}$. Let $\left(u_{k}, v_{k}\right)$ be a sequence such that $\left(u_{k}, v_{k}\right) \rightarrow$ $(u, v)$ in $L^{1}(\Omega) \times L^{1}(\Omega)$ and $\sup F_{k}\left(u_{k}, v_{k}\right)<+\infty$. Then it is straightforward that $v=1 \mathcal{L}^{n}$-a.e. in $\Omega$. We are going to show that $u \in B V(\Omega)$ and that 


$$
\Phi(u) \leq \liminf _{k \rightarrow+\infty} F_{k}\left(u_{k}, v_{k}\right),
$$

that proves the thesis under the assumption of the boundedness of $u$.

Given $\xi \in \mathbb{S}^{n-1}$, we consider a subsequence $\left(u_{r}, v_{r}\right)$ of $\left(u_{k}, v_{k}\right)$ satisfying

$$
\left(\left(u_{r}\right)_{y}^{\xi},\left(v_{r}\right)_{y}^{\xi}\right) \rightarrow\left(u_{y}^{\xi}, 1\right) \text { in } L^{1}\left(\Omega_{y}^{\xi}\right) \times L^{1}\left(\Omega_{y}^{\xi}\right) \text { for } \mathcal{H}^{n-1} \text {-a.e. } y \in \Pi^{\xi}
$$

and realizing the lower limit in (6.1) as a limit.

By Fubini's theorem and Fatou's lemma one deduces that

$$
\liminf _{r \rightarrow \infty} \int_{\Omega_{y}^{\xi}}\left(f_{r}^{2}\left(\left(v_{r}\right)_{y}^{\xi}\right)\left|\nabla\left(\left(u_{r}\right)_{y}^{\xi}\right)\right|^{2}+\frac{\left(1-\left(v_{r}\right)_{y}^{\xi}\right)^{2}}{4 \varepsilon_{r}}+\varepsilon_{r}\left|\nabla\left(\left(v_{r}\right)_{y}^{\xi}\right)\right|^{2}\right) d t<+\infty
$$

holds for $\mathcal{H}^{n-1}$-a.e. $y \in \Omega^{\xi}$.

The one-dimensional result, Proposition 5.1, yields now that $u_{y}^{\xi} \in B V\left(\Omega_{y}^{\xi}\right)$ and that

$$
\begin{aligned}
& \int_{\Omega_{y}^{\xi}} h\left(\left|\nabla\left(u_{y}^{\xi}\right)\right|\right) d t+\int_{J_{u_{y}^{\xi}}} g\left(\left|\left[u_{y}^{\xi}\right]\right|\right) d \mathcal{H}^{0}+\ell\left|D^{c} u_{y}^{\xi}\right|\left(\Omega_{y}^{\xi}\right) \leq \\
& \quad \leq \liminf _{r \rightarrow \infty} \int_{\Omega_{y}^{\xi}}\left(f_{r}^{2}\left(\left(v_{r}\right)_{y}^{\xi}\right)\left|\nabla\left(\left(u_{r}\right)_{y}^{\xi}\right)\right|^{2}+\frac{\left(1-\left(v_{r}\right)_{y}^{\xi}\right)^{2}}{4 \varepsilon_{r}}+\varepsilon_{r}\left|\nabla\left(\left(v_{r}\right)_{y}^{\xi}\right)\right|^{2}\right) d t .
\end{aligned}
$$

We first check that (6.2) implies $u \in B V(\Omega)$ by estimating $\int_{\Omega^{\xi}}\left|D\left(u_{y}^{\xi}\right)\right|\left(\Omega_{y}^{\xi}\right) d \mathcal{H}^{n-1}$. We first notice that

$$
\int_{\Omega_{y}^{\xi}}\left|\nabla\left(u_{y}^{\xi}\right)\right| d t \leq \frac{1}{\ell} \int_{\Omega_{y}^{\xi}} h\left(\left|\nabla\left(u_{y}^{\xi}\right)\right|\right) d t+\frac{\ell}{4} \mathcal{L}^{1}\left(\Omega_{y}^{\xi}\right),
$$

being $h(s) \geq \ell s-\ell^{2} / 4$.

Since $g(s) / s \rightarrow \ell$ as $s \rightarrow 0$, with fixed $\eta>0$ one has

$$
g(s)>(\ell-\eta) s \quad \text { for } s<\delta,
$$

for some $\delta$ sufficiently small.

Therefore (6.3), (6.4), and the boundedness of $u$ entail

$$
\begin{aligned}
\left|D\left(u_{y}^{\xi}\right)\right|\left(\Omega_{y}^{\xi}\right) \leq & \frac{1}{\ell} \int_{\Omega_{y}^{\xi}} h\left(\left|\nabla\left(u_{y}^{\xi}\right)\right|\right) d t+\frac{\ell}{4} \operatorname{diam} \Omega+\frac{1}{\ell-\eta} \int_{\left\{t \in J_{u_{y}^{\xi}}^{\xi}:\left|\left[u_{y}^{\xi}\right]\right|<\delta\right\}} g\left(\left|\left[u_{y}^{\xi}\right]\right|\right) d \mathcal{H}^{0} \\
& +\frac{2 M}{g(\delta)} \int_{\left\{t \in J_{u_{y}^{\xi}}:\left|\left[u_{y}^{\xi}\right]\right| \geq \delta\right\}} g\left(\left|\left[u_{y}^{\xi}\right]\right|\right) d \mathcal{H}^{0}+\left|D^{c} u_{y}^{\xi}\right|\left(\Omega_{y}^{\xi}\right) \\
\leq & +c\left(\int_{\Omega_{y}^{\xi}} h\left(\left|\nabla\left(u_{y}^{\xi}\right)\right|\right) d t+\int_{J_{u_{y}}} g\left(\left|\left[u_{y}^{\xi}\right]\right|\right) d \mathcal{H}^{0}+\ell\left|D^{c} u_{y}^{\xi}\right|\left(\Omega_{y}^{\xi}\right)\right)
\end{aligned}
$$

where diam $\Omega$ denotes the diameter of $\Omega$ and $c:=\max \left\{\frac{1}{\ell}, \frac{\ell}{4} \operatorname{diam} \Omega, \frac{1}{\ell-\eta}, \frac{2 M}{g(\delta)}\right\}$. Integrating the last inequality on $\Omega^{\xi}$ one deduces by (6.2)

$$
\int_{\Omega^{\xi}}\left|D\left(u_{y}^{\xi}\right)\right|\left(\Omega_{y}^{\xi}\right) d \mathcal{H}^{n-1} \leq c \mathcal{H}^{n-1}\left(\Omega^{\xi}\right)+c \sup _{k} F_{k}\left(u_{k}, v_{k}\right) .
$$

Taking $\xi=e_{1}, \ldots, e_{n}$ one obtains $u \in B V(\Omega)$. 
We now prove formula (6.1) using localization. The integration on $\Omega^{\xi}$ of the one-dimensional estimate in (6.2) gives

$$
\int_{\Omega} h(|\nabla u \cdot \xi|) d x+\int_{J_{u}}\left|v_{u} \cdot \xi\right| g(|[u]|) d \mathcal{H}^{n-1}+\ell \int_{\Omega}\left|\gamma_{u} \cdot \xi\right| d\left|D^{c} u\right| \leq \liminf _{k \rightarrow+\infty} F_{k}\left(u_{k}, v_{k} ; \Omega\right),
$$

where $\gamma_{u}:=\frac{d D^{c} u}{d\left|D^{c} u\right|}$ denotes the density of $D^{c} u$ with respect to $\left|D^{c} u\right|$. Let $E \subset \Omega$ be a Borel set such that $D^{a} u(E)=0$ and $D^{s} u(\Omega \backslash E)=0$, and let

$$
\lambda:=\mathcal{L}^{n}\left\llcorner\Omega \backslash E+\mathcal{H}^{n-1}\left\llcorner J_{u}+\left|D^{c} u\right|\left\llcorner E \backslash J_{u} .\right.\right.\right.
$$

Let us consider a countable dense set $D \subset \mathbb{S}^{n-1}$ and the functions

$$
\psi_{\xi}:=h(|\nabla u \cdot \xi|) \chi_{\Omega \backslash E}+\left|v_{u} \cdot \xi\right| g(|[u]|) \chi_{J_{u}}+\ell\left|\gamma_{u} \cdot \xi\right| \chi_{E \backslash J_{u}}, \quad \xi \in D .
$$

Then (6.5) gives $\left(\psi_{\xi} \lambda\right)(A) \leq F^{\prime}(u, 1, A)$ for all open sets $A \subset \Omega$. Since $F^{\prime}(u, 1, \cdot)$ is superadditive, this implies $\left(\left(\sup _{\xi} \psi_{\xi}\right) \lambda\right)(A) \leq F^{\prime}(u, 1, A)($ see $[18$, Lemma 15.2]) and therefore the conclusion.

In the general case, if $u \in L^{1} \backslash L^{\infty}(\Omega)$ one considers $\left(u_{k}^{M}, v_{k}\right)$ and $\left(u^{M}, v\right)$, where $u^{M}:=(-M \vee u) \wedge M$ denotes the truncation at level $M \in(0,+\infty)$. Since the functional $F_{k}$ decreases by truncation and $u_{k}^{M} \rightarrow u^{M}$ in $L^{1}(\Omega)$, we deduce that $u^{M} \in B V(\Omega)$ and

$$
\Phi\left(u^{M}\right) \leq \liminf _{k \rightarrow+\infty} F_{k}\left(u_{k}^{M}, v_{k}\right) \leq \liminf _{k \rightarrow+\infty} F_{k}\left(u_{k}, v_{k}\right) .
$$

Therefore $u \in G B V(\Omega)$ and (6.1) follows easily from (6.6) as $M \rightarrow+\infty$.

To prove the limsup inequality we follow an abstract approach. We first show that the $\bar{\Gamma}$-limit is a Borel measure. The only relevant property to be checked is the weak subadditivity of the $\Gamma$-limsup. This is a consequence of De Giorgi's slicing and averaging argument as shown in the following lemma.

Lemma 6.2. Let $(u, v) \in L^{1}(\Omega) \times L^{1}(\Omega)$, let $A^{\prime}, A, B \in \mathcal{A}(\Omega)$ with $A^{\prime} \subset \subset A$, then

$$
F^{\prime \prime}\left(u, 1 ; A^{\prime} \cup B\right) \leq F^{\prime \prime}(u, 1 ; A)+F^{\prime \prime}(u, 1 ; B),
$$

where $F^{\prime \prime}$ is the $\Gamma$-limsup of the sequence $F_{k}$ defined in (3.1).

Proof. We assume that the right-hand side of (6.7) is finite, so that $u \in G B V(A \cup B)$ and $v=1 \mathcal{L}^{n}$-a.e. in $A \cup B$. We can reduce the problem to the case of functions $u \in B V \cap L^{\infty}(A \cup B)$. This is a straightforward consequence of the fact that the energies $F_{k}$ 's, and thus the $\Gamma$-limsup $F^{\prime \prime}$, are decreasing by truncations. Actually, thanks to $L^{1}$ lower semicontinuity, they are continuous under such an operation.

Under this assumption, let $\left(u_{k}^{A}, v_{k}^{A}\right),\left(u_{k}^{B}, v_{k}^{B}\right)$ be recovery sequences for $(u, 1)$ on $A$ and $B$ respectively, that is,

$$
\left(u_{k}^{A}, v_{k}^{A}\right),\left(u_{k}^{B}, v_{k}^{B}\right) \rightarrow(u, 1) \text { in } L^{1}(\Omega) \times L^{1}(\Omega),
$$

and

$$
\limsup _{k \rightarrow+\infty} F_{k}\left(u_{k}^{A}, v_{k}^{A} ; A\right)=F^{\prime \prime}(u, 1 ; A), \quad \limsup _{k \rightarrow+\infty} F_{k}\left(u_{k}^{B}, v_{k}^{B} ; B\right)=F^{\prime \prime}(u, 1 ; B) .
$$

Note that, again up to truncations, we may assume that

$$
\left(u_{k}^{A}, v_{k}^{A}\right),\left(u_{k}^{B}, v_{k}^{B}\right) \quad \text { are bounded in } L^{\infty}(\Omega) .
$$

To simplify the calculations below we introduce the functionals $G_{k}: L^{1}(\Omega) \times \mathcal{A}(\Omega) \rightarrow[0,+\infty]$ given by

$$
G_{k}(v ; O):=\int_{O}\left(\frac{(1-v)^{2}}{4 \varepsilon_{k}}+\varepsilon_{k}|\nabla v|^{2}\right) d x, \quad \text { if } v \in H^{1}(\Omega),
$$

$+\infty$ otherwise. Notice that 


$$
F_{k}(u, v ; O)=\int_{O} f_{k}^{2}(v)|\nabla u|^{2} d x+G_{k}(v ; O) .
$$

Let $\delta:=\operatorname{dist}\left(A^{\prime}, \partial A\right)>0$, and with fixed $M \in \mathbb{N}$, we set for all $i \in\{1, \ldots, M\}$

$$
A_{i}:=\left\{x \in \Omega: \operatorname{dist}\left(x, A^{\prime}\right)<\frac{\delta}{M} i\right\},
$$

and $A_{0}:=A^{\prime}$. Clearly, we have $A_{i-1} \subset \subset A_{i} \subset A$. Denote by $\varphi_{i} \in C_{c}^{1}(\Omega)$ a cut-off function between $A_{i-1}$ and $A_{i}$, i.e., $\left.\varphi_{i}\right|_{A_{i-1}}=1,\left.\varphi_{i}\right|_{A_{i}^{c}}=0$, and $\left\|\nabla \varphi_{i}\right\|_{L^{\infty}(\Omega)} \leq \frac{2 M}{\delta}$. Then, set

$$
u_{k}^{i}:=\varphi_{i} u_{k}^{A}+\left(1-\varphi_{i}\right) u_{k}^{B},
$$

and

$$
v_{k}^{i}:= \begin{cases}\varphi_{i-1} v_{k}^{A}+\left(1-\varphi_{i-1}\right)\left(v_{k}^{A} \wedge v_{k}^{B}\right) & \text { on } A_{i-1} \\ v_{k}^{A} \wedge v_{k}^{B} & \text { on } A_{i} \backslash A_{i-1} \\ \varphi_{i+1}\left(v_{k}^{A} \wedge v_{k}^{B}\right)+\left(1-\varphi_{i+1}\right) v_{k}^{B} & \text { on } \Omega \backslash A_{i} .\end{cases}
$$

With fixed $i \in\{2, \ldots, M-1\},\left(u_{k}^{i}, v_{k}^{i}\right) \in H^{1}(\Omega) \times H^{1}(\Omega)$ and their energy on $A^{\prime} \cup B$ can be estimated by

$$
F_{k}\left(u_{k}^{i}, v_{k}^{i} ; A^{\prime} \cup B\right) \leq F_{k}\left(u_{k}^{A}, v_{k}^{A} ; A_{i-2}\right)+F_{k}\left(u_{k}^{B}, v_{k}^{B} ; B \backslash A_{i+1}\right)+F_{k}\left(u_{k}^{i}, v_{k}^{i} ; B \cap\left(A_{i+1} \backslash A_{i-2}\right)\right) \text {. }
$$

Therefore, we need to bound only the last term. To this end we further split the contributions in each layer; in estimating each of such terms we shall repeatedly use the monotonicity of $f_{k}$ and the fact that it is bounded by 1 . In addition, a positive constant, which may vary from line to line, will appear in the formulas below. Elementary computations and the definitions in (6.11) and (6.12) give, using $v_{k}^{i} \leq v_{k}^{A}$,

$$
\begin{aligned}
F_{k}\left(u_{k}^{i}, v_{k}^{i} ; B \cap\left(A_{i-1} \backslash A_{i-2}\right)\right) \leq & \int_{B \cap\left(A_{i-1} \backslash A_{i-2}\right)} f_{k}^{2}\left(v_{k}^{A}\right)\left|\nabla u_{k}^{A}\right|^{2} d x+G_{k}\left(v_{k}^{i} ; B \cap\left(A_{i-1} \backslash A_{i-2}\right)\right) \\
\leq & c\left(F_{k}\left(u_{k}^{A}, v_{k}^{A} ; B \cap\left(A_{i-1} \backslash A_{i-2}\right)\right)+F_{k}\left(u_{k}^{B}, v_{k}^{B} ; B \cap\left(A_{i-1} \backslash A_{i-2}\right)\right)\right) \\
& +\frac{c M^{2} \varepsilon_{k}}{\delta^{2}} \int_{B \cap\left(A_{i-1} \backslash A_{i-2}\right)}\left|v_{k}^{A}-v_{k}^{B}\right|^{2} d x,
\end{aligned}
$$

$$
\begin{aligned}
& F_{k}\left(u_{k}^{i}, v_{k}^{i} ; B \cap\left(A_{i} \backslash A_{i-1}\right)\right) \\
& \quad \leq c \int_{B \cap\left(A_{i} \backslash A_{i-1}\right)} f_{k}^{2}\left(v_{k}^{A} \wedge v_{k}^{B}\right)\left(\left|\nabla u_{k}^{A}\right|^{2}+\left|\nabla u_{k}^{B}\right|^{2}+\frac{4 M^{2}}{\delta^{2}}\left|u_{k}^{A}-u_{k}^{B}\right|^{2}\right) d x+G_{k}\left(v_{k}^{A} \wedge v_{k}^{B} ; B \cap\left(A_{i} \backslash A_{i-1}\right)\right) \\
& \quad \leq c\left(F_{k}\left(u_{k}^{A}, v_{k}^{A} ; B \cap\left(A_{i} \backslash A_{i-1}\right)\right)+F_{k}\left(u_{k}^{B}, v_{k}^{B} ; B \cap\left(A_{i} \backslash A_{i-1}\right)\right)\right)+\frac{c M^{2}}{\delta^{2}} \int_{B \cap\left(A_{i} \backslash A_{i-1}\right)}\left|u_{k}^{A}-u_{k}^{B}\right|^{2} d x,
\end{aligned}
$$

and

$$
\begin{aligned}
F_{k}\left(u_{k}^{i}, v_{k}^{i} ; B \cap\left(A_{i+1} \backslash A_{i}\right)\right) \leq & \int_{B \cap\left(A_{i+1} \backslash A_{i}\right)} f_{k}^{2}\left(v_{k}^{B}\right)\left|\nabla u_{k}^{B}\right|^{2} d x+G_{k}\left(v_{k}^{i} ; B \cap\left(A_{i+1} \backslash A_{i}\right)\right) \\
\leq & c\left(F_{k}\left(u_{k}^{A}, v_{k}^{A} ; B \cap\left(A_{i+1} \backslash A_{i}\right)\right)+F_{k}\left(u_{k}^{B}, v_{k}^{B} ; B \cap\left(A_{i+1} \backslash A_{i}\right)\right)\right) \\
& +\frac{c M^{2} \varepsilon_{k}}{\delta^{2}} \int_{B \cap\left(A_{i+1} \backslash A_{i}\right)}\left|v_{k}^{A}-v_{k}^{B}\right|^{2} d x .
\end{aligned}
$$

By adding (6.13)-(6.14), we deduce that 


$$
\begin{aligned}
F_{k}\left(u_{k}^{i}, v_{k}^{i} ; A^{\prime} \cup B\right) \leq & F_{k}\left(u_{k}^{A}, v_{k}^{A} ; A\right)+F_{k}\left(u_{k}^{B}, v_{k}^{B} ; B\right) \\
& +c\left(F_{k}\left(u_{k}^{A}, v_{k}^{A} ; B \cap\left(A_{i+1} \backslash A_{i-2}\right)\right)+F_{k}\left(u_{k}^{B}, v_{k}^{B} ; B \cap\left(A_{i+1} \backslash A_{i-2}\right)\right)\right) \\
& +\frac{c M^{2}}{\delta^{2}} \int_{B \cap\left(A_{i+1} \backslash A_{i-2}\right)}\left|u_{k}^{A}-u_{k}^{B}\right|^{2} d x+\frac{c M^{2} \varepsilon_{k}}{\delta^{2}} \int_{B \cap\left(A_{i+1} \backslash A_{i-2}\right)}\left|v_{k}^{A}-v_{k}^{B}\right|^{2} d x .
\end{aligned}
$$

Hence, by summing up on $i \in\{2, \ldots, M-1\}$ and taking the average, for each $k$ we may find an index $i_{k}$ in that range such that

$$
\begin{aligned}
F_{k}\left(u_{k}^{i_{k}}, v_{k}^{i_{k}} ; A^{\prime} \cup B\right) \leq & F_{k}\left(u_{k}^{A}, v_{k}^{A} ; A\right)+F_{k}\left(u_{k}^{B}, v_{k}^{B} ; B\right) \\
& +\frac{c}{M}\left(F_{k}\left(u_{k}^{A}, v_{k}^{A} ; B \cap\left(A \backslash A^{\prime}\right)\right)+F_{k}\left(u_{k}^{B}, v_{k}^{B} ; B \cap\left(A \backslash A^{\prime}\right)\right)\right) \\
& +\frac{c M}{\delta^{2}} \int_{B \cap\left(A \backslash A^{\prime}\right)}\left|u_{k}^{A}-u_{k}^{B}\right|^{2} d x+\frac{c M \varepsilon_{k}}{\delta^{2}} \int_{B \cap\left(A \backslash A^{\prime}\right)}\left|v_{k}^{A}-v_{k}^{B}\right|^{2} d x .
\end{aligned}
$$

By (6.8) we deduce that $\left(u_{k}^{i_{k}}, v_{k}^{i_{k}}\right) \rightarrow(u, 1)$ in $L^{1}(\Omega) \times L^{1}(\Omega)$, and actually in $L^{q}(\Omega) \times L^{q}(\Omega)$ for all $q \in[1,+\infty)$ thanks to the uniform boundedness assumption in (6.10). Therefore, in view of (6.9) and the definition of $\Gamma$-limsup we infer that

$$
F^{\prime \prime}\left(u, 1 ; A^{\prime} \cup B\right) \leq\left(1+\frac{c}{M}\right)\left(F^{\prime \prime}(u, 1 ; A)+F^{\prime \prime}(u, 1 ; B)\right) .
$$

The conclusion then follows by passing to the limit on $M \uparrow \infty$.

We next prove that $F^{\prime \prime}(u, 1 ; \cdot)$ is controlled in terms of the Mumford-Shah functional $M S$, whose definition is given in (2.1). This result gives a first rough estimate for the upper bound inequality. We shall improve on the jump part in Proposition 6.4 below and finally we shall conclude the proof of the $\Gamma$-limsup inequality using a relaxation argument.

Lemma 6.3. For all $u \in L^{1}(\Omega)$ and $A \in \mathcal{A}(\Omega)$ it holds

$$
F^{\prime \prime}(u, 1 ; A) \leq M S(u ; A),
$$

where $F^{\prime \prime}$ is the $\Gamma$-limsup of the sequence $F_{k}$ defined in (3.1) and MS is introduced in (2.1).

Proof. Denote by $\psi:[0,1] \rightarrow[0,1]$ any nondecreasing lower-semicontinuous function such that $\psi^{-1}(0)=0$, $\psi(1)=1$ and

$$
\sup _{k} f_{k}(z) \leq \psi(z) \quad \text { for all } z \in[0,1]
$$

for instance $\psi=\chi_{(0,1]}$ satisfies all the conditions written above. Consider the corresponding functionals $A T_{k}^{\psi}: L^{1}(\Omega) \times L^{1}(\Omega) \rightarrow[0,+\infty]$ defined in (2.2), and note that $F_{k} \leq A T_{k}^{\psi}$ for every $k$. The upper bound inequality for $\left(F_{k}\right)$ then follows at once from the classical results by Ambrosio and Tortorelli (cp. [9], and see also [30]).

We are now ready to prove the upper bound inequality.

Proposition 6.4. Let $F$ be the functional introduced in (3.9). For every $(u, v) \in L^{1}(\Omega) \times L^{1}(\Omega)$ it holds

$$
F^{\prime \prime}(u, v) \leq F(u, v),
$$

where $F^{\prime \prime}$ is the $\Gamma$-limsup of the sequence $F_{k}$ defined in (3.1).

Proof. Since $L^{1}$ is separable, given any subsequence $\left(F_{k_{j}}\right)$ of $\left(F_{k}\right)$ we may extract a further subsequence, not relabeled for convenience, $\bar{\Gamma}$-converging to some $\widehat{F}$ (see [25, Theorem 16.9]).

The functional $\widehat{F}(u, v ; \cdot)$ is by definition increasing and inner regular. Since $F_{k}(u, v ; \cdot)$ is additive, one easily deduces that $F^{\prime}$ is superadditive and from this that its inner regular envelope $\widehat{F}=\left(F^{\prime}\right)_{-}$is superadditive (see [25, Proposition 14.18 or Proposition 16.12]). 
Using Lemma 6.2 one can show that $\widehat{F}=\left(F^{\prime \prime}\right)_{-}$is subadditive (see [25, Lemma 14.20 and the proof of Proposition 18.4]). Therefore $\widehat{F}$ is the restriction to open sets of the Borel measure

$$
F_{*}(u, v ; E)=\inf \{\widehat{F}(u, v ; A): A \in \mathcal{A}(\Omega) ; E \subset A\},
$$

see [25, Theorem 14.23], in the following we identify $\widehat{F}$ and $F_{*}$.

If $u \in L^{1}(\Omega)$ is such that $M S(u ; \Omega)<+\infty$, then by Lemma 6.3 we obtain $F^{\prime \prime}(u, 1 ; \cdot) \leq M S(u ; \cdot)<+\infty$ on all open sets, and by the regularity properties of Radon measures $F^{\prime \prime}$ coincides with its inner envelope. Indeed, for a given open set $A$ and $\varepsilon>0$, choose open sets $A^{\prime}, A^{\prime \prime}$ and $C$ with $A^{\prime} \subset \subset A^{\prime \prime} \subset \subset A$ and $A \backslash A^{\prime} \subset C$ such that $M S(u ; C) \leq \varepsilon$. Then use Lemmas 6.2 and 6.3 to estimate $F^{\prime \prime}(u, 1 ; A) \leq F^{\prime \prime}\left(u, 1 ; A^{\prime} \cup C\right) \leq F^{\prime \prime}\left(u, 1 ; A^{\prime \prime}\right)+M S(u ; C) \leq F^{\prime \prime}\left(u, 1 ; A^{\prime \prime}\right)+\varepsilon$. In other words, $\widehat{F}(u, 1)$ is the $\Gamma$-limit of $F_{k_{j}}$ for all $u$ such that $M S(u)<+\infty$.

For all $u \in S B V^{2}(\Omega)$ in particular the estimate in Lemma 6.3 implies that

$$
\widehat{F}\left(u, 1 ; \Omega \backslash J_{u}\right) \leq \int_{\Omega}|\nabla u|^{2} d x .
$$

We provide below for the same $u$ the estimate

$$
\widehat{F}\left(u, 1 ; J_{u}\right) \leq \int_{J_{u}} g(|[u]|) d \mathcal{H}^{n-1} .
$$

Given this for granted we conclude as follows: we consider the functional $F_{\infty}: B V(\Omega) \rightarrow[0,+\infty]$

$$
F_{\infty}(u):= \begin{cases}\int_{\Omega}|\nabla u|^{2} d x+\int_{J_{u}} g(|[u]|) d \mathcal{H}^{n-1} & \text { if } u \in S B V^{2}(\Omega) \\ +\infty & \text { otherwise on } B V(\Omega) .\end{cases}
$$

Further, note that by [13, Theorem 3.1 and Propositions 3.3-3.5] its relaxation w.r.t. the $w *-B V$ topology is given on $B V(\Omega)$ by $F(\cdot, 1)$. By $(6.15)$ and $(6.16)$ we have that $\widehat{F} \leq F_{\infty}$, and since $\widehat{F}(\cdot, 1)$ is $L^{1}$-lower semicontinuous, we infer that

$$
\widehat{F}(u, 1) \leq F(u, 1) \quad \text { for all } u \in B V(\Omega) .
$$

We conclude that the same inequality is true for all $u \in G B V \cap L^{1}(\Omega)$ by the usual truncation argument. Finally, combining the latter estimate with the lower estimate of Proposition 6.1 allows us to deduce that the $\Gamma$-limit does not depend on the chosen subsequence and it is equal to $F$. Hence, by Urysohn's property the whole family $\left(F_{k}\right)$ $\Gamma$-converges to $F$ (cp. [25, Proposition 8.3]).

Let us now prove formula (6.16). To this end, fixed $\lambda>0$ we introduce the perturbed functional

$$
\widehat{F_{\lambda}}(u, 1):=\widehat{F}(u, 1)+\lambda\left(\int_{\Omega}|\nabla u|^{2} d x+\int_{J_{u}}(1+|[u]|) d \mathcal{H}^{n-1}\right)
$$

for all $u \in S B V^{2}(\Omega)$. We may apply to $\widehat{F_{\lambda}}$ the integral representation result [14, Theorem 1] to infer that for $\mathcal{H}^{n-1}$-a.e. $x \in J_{u}$

$$
\begin{array}{r}
\frac{d \widehat{F_{\lambda}}(u, 1 ; \cdot)}{d\left(\mathcal{H}^{n-1}\left\llcorner J_{u}\right)\right.}(x)=\limsup _{\delta \downarrow 0} \frac{1}{\delta^{n-1}} \inf \left\{\widehat{F_{\lambda}}\left(w, 1 ; x+\delta Q_{v_{u}(x)}\right): w \in S B V^{2}\left(x+\delta Q_{v_{u}(x)}\right),\right. \\
\left.w=u_{x} \text { on a neighborhood of } x+\delta \partial Q_{v_{u}(x)}\right\},
\end{array}
$$

where

$$
u_{x}(y):= \begin{cases}u^{+}(x) & \text { if }\left\langle y-x, v_{u}(x)\right\rangle>0 \\ u^{-}(x) & \text { if }\left\langle y-x, v_{u}(x)\right\rangle<0\end{cases}
$$

and $Q_{v_{u}(x)}$ denotes any cube of side 1 centered in the origin and with a face orthogonal to $v_{u}(x)$. Hence, it is enough to show that for $\mathcal{H}^{n-1}$-a.e. $x \in J_{u}$ 


$$
\underset{\delta \downarrow 0}{\limsup } \frac{1}{\delta^{n-1}} \widehat{F}\left(u_{x}, 1 ; x+\delta Q_{v_{u}(x)}\right) \leq g(|[u](x)|),
$$

since by taking $u_{x}$ itself as test function in (6.17) we get

$$
\frac{d \widehat{F_{\lambda}}(u, 1 ; \cdot)}{d\left(\mathcal{H}^{n-1}\left\llcorner J_{u}\right)\right.}(x) \leq \limsup _{\delta \downarrow 0} \frac{1}{\delta^{n-1}} \widehat{F}\left(u_{x}, 1 ; x+\delta Q_{v_{u}(x)}\right)+\lambda(1+|[u](x)|),
$$

in turn implying

$$
\widehat{F}\left(u, 1 ; J_{u}\right) \leq \widehat{F_{\lambda}}\left(u, 1 ; J_{u}\right) \leq \int_{J_{u}}(g(|[u](x)|)+\lambda+\lambda|[u](x)|) d \mathcal{H}^{n-1} .
$$

Finally, (6.16) follows at once by letting $\lambda \downarrow 0$.

Formula (6.18) easily follows by repeating the one-dimensional construction of Proposition 5.2. More precisely, assume $x=0$ and $v_{u}(x)=e_{n}$ for simplicity. With fixed $\eta>0$, let $T_{\eta}>0$ and $\alpha_{\eta}, \beta_{\eta} \in H^{1}\left(\left(0, T_{\eta}\right)\right)$ be such that $\alpha_{\eta}(0)=u^{-}(0), \alpha_{\eta}\left(T_{\eta}\right)=u^{+}(0), \beta_{\eta}(0)=\beta_{\eta}\left(T_{\eta}\right)=1, u^{-}(0) \leq \alpha_{\eta} \leq u^{+}(0), 0 \leq \beta_{\eta} \leq 1$, and

$$
\int_{0}^{T_{\eta}}\left(f^{2}\left(\beta_{\eta}\right)\left|\alpha_{\eta}^{\prime}\right|^{2}+\frac{\left(1-\beta_{\eta}\right)^{2}}{4}+\left|\beta_{\eta}^{\prime}\right|^{2}\right) d t \leq g(|[u](0)|)+\eta .
$$

Let $A_{j}:=\left(-\frac{\varepsilon_{k_{j}} T_{\eta}}{2}, \frac{\varepsilon_{k_{j}} T_{\eta}}{2}\right)$, and set

$$
\begin{aligned}
& u_{j}(y):= \begin{cases}\alpha_{\eta}\left(\frac{y_{n}}{\varepsilon_{k_{j}}}+\frac{T_{\eta}}{2}\right) & \text { if } y_{n} \in A_{j} \\
u_{0} & \text { otherwise, }\end{cases} \\
& v_{j}(y):= \begin{cases}\beta_{\eta}\left(\frac{y_{n}}{\varepsilon_{k_{j}}}+\frac{T_{\eta}}{2}\right) & \text { if } y_{n} \in A_{j} \\
1 & \text { otherwise. }\end{cases}
\end{aligned}
$$

Clearly, $\left(u_{j}, v_{j}\right) \rightarrow\left(u_{0}, 1\right)$ in $L^{1}\left(Q_{e_{n}}\right) \times L^{1}\left(Q_{e_{n}}\right)$, and if $Q_{e_{n}}^{\prime}=Q_{e_{n}} \cap\left(\mathbb{R}^{n-1} \times\{0\}\right)$, a change of variable yields

$$
\begin{aligned}
F_{k_{j}}\left(u_{j}, v_{j} ; \delta Q_{e_{n}}\right) & =F_{k_{j}}\left(u_{j}, v_{j}, \delta Q_{e_{n}}^{\prime} \times A_{j}\right) \\
& \leq \delta^{n-1} \int_{0}^{T_{\eta}}\left(f^{2}\left(\beta_{\eta}\right)\left|\alpha_{\eta}^{\prime}\right|^{2}+\frac{\left(1-\beta_{\eta}\right)^{2}}{4}+\left|\beta_{\eta}^{\prime}\right|^{2}\right) d t \leq \delta^{n-1}(g(|[u](0)|)+\eta) .
\end{aligned}
$$

Therefore, by the very definition of $\widehat{F}$ we infer that

$$
\widehat{F}\left(u_{0}, 1 ; \delta Q_{e_{n}}\right) \leq \delta^{n-1}(g(|[u](0)|)+\eta),
$$

and estimate (6.18) follows at once dividing by $\delta^{n-1}$, taking the superior limit as $\delta \downarrow 0$, and finally by letting $\eta \downarrow 0$ in the formula above.

The proof of the compactness result, Theorem 3.3, follows the lines of [26, Theorem 7.4], so we just sketch the relevant arguments and refer to [26] for more details.

Proof of Theorem 3.3. Let us start assuming that $n=1$ and $M:=\sup _{k}\left\|u_{k}\right\|_{L^{\infty}(\Omega)}<+\infty, \Omega=(0,1)$. Repeating the proof of Theorem 5.1 one finds that $v_{k} \rightarrow 1$ in $L^{1}(\Omega)$ and that for every $\delta>0$ there exists a finite subset $S \subset \Omega$ for which

$$
(1-\omega(\delta)) \int_{\Omega \backslash S_{\eta}} h\left(\left|u_{k}^{\prime}\right|\right) d x \leq F_{k}\left(u_{k}, v_{k}, \Omega \backslash S_{\eta}\right)
$$


holds for $\eta>0$ small (dependently on $\delta$ ) and for $k$ large (dependently on $\eta$ ), where $\omega(\delta) \rightarrow 0$ for $\delta \rightarrow 0$, and $S_{\eta}:=$ $\bigcup_{i=1}^{L}\left(t_{i}-\eta, t_{i}+\eta\right)$. This implies that $u_{k}$ is bounded in $B V\left(\Omega \backslash S_{\eta}\right)$ uniformly with respect to $k$ and $\eta$. Hence up to subsequences $u_{k}$ converges to a function $u \in B V\left(\Omega \backslash S_{\eta}\right) \mathcal{L}^{1}$-a.e. in $\Omega \backslash S_{\eta}$. The boundedness hypothesis and a diagonalization argument yield that $u$ in fact belongs to $B V(\Omega)$ and that $u_{k} \rightarrow u$ in $L^{1}(\Omega)$.

For $n>1$, we fix $\xi \in \mathbb{S}^{n-1}$ and $\delta>0$ and introduce the sequence $w_{k}$ whose slices satisfy

$$
\begin{aligned}
& \left(w_{k}\right)_{y}^{\xi}:= \begin{cases}\left(u_{k}\right)_{y}^{\xi} & \text { if } y \in A_{k}, \\
0 & \text { otherwise, }\end{cases} \\
& A_{k}:=\left\{y \in \Omega^{\xi}: F_{k}^{1}\left(\left(u_{k}\right)_{y}^{\xi},\left(v_{k}\right)_{y}^{\xi}\right) \leq M / \delta^{2}\right\},
\end{aligned}
$$

where $F_{k}^{1}$ denotes the one-dimensional counterpart of the functional $F_{k}$. Then $w_{k}$ is bounded in $L^{\infty}(\Omega), u_{k}$ is in a $\delta$-neighborhood of $w_{k}$ in $L^{1}(\Omega)$, and the $n=1$ case shows that $\left(w_{k}\right)_{y}^{\xi}$ is pre-compact in $L^{1}(\Omega)$. Then the precompactness of $u_{k}$ in $L^{1}(\Omega)$ is ensured by [1, Theorem 6.6] as $\xi$ varies in a basis of $\mathbb{R}^{n}$.

Finally, the unbounded case is done by truncation as in [26, Theorem 7.4].

\section{Further results}

In this section we build upon the results in Sections 3-6 to obtain in the limit different models by slightly changing the approximating energies $F_{k}$ 's. More precisely, we shall approximate a cohesive model with Dugdale's surface density, a cohesive model with power-law growth at small openings, and a model in Griffith's brittle fracture.

This task will be accomplished by letting the function $f$ vary as in item (ii) of Proposition 4.5 in the first instance, as in item (iii) in the third, and suitably in the second (cp. (iii) of Proposition 7.3 below), respectively. More precisely, we consider a sequence of functions $\left(f^{(j)}\right)$ satisfying (3.3) and (3.4) and for all $j, k \in \mathbb{N}$ introduce the energies

$$
F_{k}^{(j)}(u, v):= \begin{cases}\int_{\Omega}\left(\left(f_{k}^{(j)}\right)^{2}(v)|\nabla u|^{2}+\frac{(1-v)^{2}}{4 \varepsilon_{k}}+\varepsilon_{k}|\nabla v|^{2}\right) d x & \text { if }(u, v) \in H^{1}(\Omega) \times H^{1}(\Omega) \\ +\infty & \text { and } 0 \leq v \leq 1 \mathcal{L}^{n} \text {-a.e. in } \Omega, \\ \text { otherwise, }\end{cases}
$$

where $f_{k}^{(j)}(z):=1 \wedge \varepsilon_{k}^{\frac{1}{2}} f^{(j)}(z)$.

In each of Theorems 7.1, 7.4, and 7.5 below we shall further specify the nature of the sequence $\left(f^{(j)}\right)$.

\subsection{Dugdale's cohesive model}

In order to approximate Dugdale's model $\mathscr{D}: L^{1}(\Omega) \rightarrow[0,+\infty]$

$$
\mathscr{D}(u):= \begin{cases}\int_{\Omega} h(|\nabla u|) d x+\int_{J_{u}}(1 \wedge \ell|[u]|) d \mathcal{H}^{n-1}+\ell\left|D^{c} u\right|(\Omega) & \text { if } u \in G B V(\Omega), \\ +\infty & \text { otherwise, }\end{cases}
$$

with $h$ as in (3.6), we shall consider the specific choice

$$
f^{(j)}(z):=\left(a_{j} z\right) \vee f(z)
$$

with $f$ satisfying (3.3) and (3.4), and

$$
\left(a_{j}\right) \text { nondecreasing, } a_{j} \uparrow \infty \text { and such that } a_{j} \varepsilon_{j}^{\frac{1}{2}} \downarrow 0 \text {. }
$$

Theorem 7.1. Suppose that $\left(f^{(j)}\right)$ is as in (7.2) and (7.3) above.

Then, the functionals $F_{k}^{(k)}$ from (7.1) $\Gamma$-converge in $L^{1}(\Omega) \times L^{1}(\Omega)$ to the functional $\widetilde{D}$ defined as follows

$$
\widetilde{\mathscr{D}}(u, v):= \begin{cases}\mathscr{D}(u) & \text { if } v=1 \mathcal{L}^{n} \text {-a.e. in } \Omega, \\ +\infty & \text { otherwise. }\end{cases}
$$


Proof. The definitions in (3.1) and (7.1) give $F_{k}^{(j)} \leq F_{k}^{(k)}$ for $j \leq k$, being $\left(f^{(j)}\right)$ nondecreasing by assumption. Hence, by Theorem 3.1 we deduce

$$
\Gamma-\liminf _{k} F_{k}^{(k)}(u, v) \geq F^{(j)}(u, v),
$$

where $F^{(j)}$ is defined as $F$ in (3.9) with $f$ substituted by $f^{(j)}$ in formulas (3.4) and (3.6) defining the volume density, and (3.7) defining the surface density.

In particular, being $\ell_{j}=\ell$ for all $j$, the corresponding volume density $h_{j}$ equals the function $h$ in (3.6). Moreover, the surface energy densities $g_{j}$ are dominated by the constant 1, and by item (ii) in Proposition 4.5 we have $\lim _{j} g_{j}(s)=1 \wedge \ell s$ for all $s \in[0,+\infty)$. In conclusion, if $\Gamma-\liminf _{k} F_{k}^{(k)}(u, v)<+\infty$, we infer that $v=1 \mathcal{L}^{n}$-a.e. in $\Omega, u \in G B V(\Omega)$, and

$$
\Gamma-\liminf _{k} F_{k}^{(k)}(u, 1) \geq \mathscr{D}(u),
$$

by the dominated convergence theorem, as $j \uparrow \infty$ in (7.4).

The upper bound inequality follows by arguing as in Proposition 6.4. Indeed, we first note that by a careful inspection of the proofs, Lemmas 6.2 and 6.3 are still valid in this generalized framework. More precisely, Lemma 6.2 continue to hold true as we have only used that each function $f_{k}=1 \wedge \varepsilon_{k}^{\frac{1}{2}} f$ in (3.2) is nondecreasing and bounded by $\chi_{(0,1]}$, properties enjoyed by $f_{k}^{(k)}$ as well.

In conclusion, as a first step we establish the estimate

$$
\limsup _{\delta \downarrow 0} \frac{1}{\delta^{n-1}} \widehat{F}\left(u_{x}, 1 ; x+\delta Q_{v_{u}(x)}\right) \leq 1 \wedge \ell|[u](x)|,
$$

for $u \in S B V^{2}(\Omega)$ and for $\mathcal{H}^{n-1}$-a.e. $x \in J_{u}$, where $\widehat{F}$ is the $\bar{\Gamma}$-limit of a properly chosen subsequence $\left(F_{k_{j}}^{\left(k_{j}\right)}\right)$ of $\left(F_{k}^{(k)}\right)$ (cp. Proposition 6.4).

Given (7.5), the derivation of the upper bound inequality in general follows exactly as in Proposition 6.4.

Let us now prove (7.5) by means of a one-dimensional construction. For the sake of simplicity we assume $x=0$ and $v_{u}(x)=e_{n}$. Actually, in view of the estimate in Lemma 6.3 we need only to discuss the case $|[u](0)|<\ell^{-1}$. To this end, set

$$
z_{j}:=\sup \left\{z \in[0,1): a_{k_{j}} z=f^{(j)}(z)\right\},
$$

it is easy to check that $z_{j}$ is actually a maximum, i.e., $a_{k_{j}} z_{j}=f^{(j)}\left(z_{j}\right)$, and that $z_{j} \leq z_{j+1}<1$ with $z_{j} \uparrow 1$. Let now

$$
T_{j}:=|[u](0)| \frac{f\left(z_{j}\right)}{1-z_{j}},
$$

then $T_{j} \uparrow \infty$. Define $\alpha_{j}(t):=u^{-}(0)$ on $\left[-T_{j}-1,-T_{j}\right], \alpha_{j}(t):=[u](0) \cdot\left(\frac{t}{2 T_{j}}+\frac{1}{2}\right)+u^{-}(0)$ on $\left[-T_{j}, T_{j}\right], \alpha_{j}(t):=$ $u^{+}(0)$ on $\left[T_{j}, T_{j}+1\right]$, and $\beta_{j}(t):=z_{j}$ on $\left[-T_{j}, T_{j}\right], \beta_{j}(t):=\left(1-z_{j}\right)\left(|t|-T_{j}\right)+z_{j}$ otherwise in $\left[-T_{j}-1, T_{j}+1\right]$.

Setting $A_{j}:=\left(-\varepsilon_{k_{j}}\left(T_{j}+1\right), \varepsilon_{k_{j}}\left(T_{j}+1\right)\right)$, we have that $\mathcal{L}^{1}\left(A_{j}\right) \rightarrow 0$ as $j \uparrow \infty$ by (7.3). Indeed, in view of (3.4) and the definition of $z_{j}$ it is easy to deduce that $\left(1-z_{j}\right) a_{k_{j}} \rightarrow \ell$ as $j \uparrow \infty$, so that $\varepsilon_{k_{j}} T_{j} \sim \varepsilon_{k_{j}} a_{k_{j}}^{2} \rightarrow 0$ as $j \uparrow \infty$ thanks to (7.3). Therefore, if

$$
\begin{aligned}
& u_{j}(y):= \begin{cases}\alpha_{j}\left(\frac{y_{n}}{\varepsilon_{k_{j}}}\right) & \text { if } y_{n} \in A_{j} \\
u_{0} & \text { otherwise, }\end{cases} \\
& v_{j}(y):= \begin{cases}\beta_{j}\left(\frac{y_{n}}{\varepsilon_{k_{j}}}\right) & \text { if } y_{n} \in A_{j} \\
1 & \text { otherwise }\end{cases}
\end{aligned}
$$

then $\left(u_{j}, v_{j}\right) \rightarrow\left(u_{0}, 1\right)$ on $L^{1}\left(Q_{e_{n}}\right) \times L^{1}\left(Q_{e_{n}}\right)$, where $u_{0}=u^{-}(0) \chi_{\left\{y_{n} \leq 0\right\}}+u^{+}(0) \chi_{\left\{y_{n}>0\right\}}$. 
Moreover, if $Q_{e_{n}}^{\prime}=Q_{e_{n}} \cap\left(\mathbb{R}^{n-1} \times\{0\}\right)$, then a change of variable yields

$$
\begin{aligned}
& F_{k_{j}}^{\left(k_{j}\right)}\left(u_{j}, v_{j} ; \delta Q_{e_{n}}\right) \\
& \quad=F_{k_{j}}^{\left(k_{j}\right)}\left(u_{j}, v_{j} ; \delta Q_{e_{n}}^{\prime} \times A_{j}\right) \\
& \quad \leq \delta^{n-1}\left(\int_{-T_{j}}^{T_{j}}\left(f^{2}\left(\beta_{j}\right)\left|\nabla \alpha_{j}\right|^{2}+\frac{\left(1-\beta_{j}\right)^{2}}{4}\right) d t+2 \int_{T_{j}}^{T_{j}+1}\left(\frac{\left|1-\beta_{j}\right|^{2}}{4}+\left|\beta_{j}^{\prime}\right|^{2}\right) d t\right) \\
& =\delta^{n-1}\left(\left(f^{2}\left(z_{j}\right) \frac{|[u](0)|^{2}}{2 T_{j}}+2 T_{j} \frac{\left(1-z_{j}\right)^{2}}{4}\right)+2\left(1-z_{j}\right)^{2} \int_{T_{j}}^{T_{j}+1} \frac{\left(t-\left(T_{j}+1\right)\right)^{2}}{4} d t+2\left(1-z_{j}\right)^{2}\right) \\
& =\delta^{n-1}\left(\left(1-z_{j}\right) f\left(z_{j}\right)|[u](0)|+\frac{13}{6}\left(1-z_{j}\right)^{2}\right)=\delta^{n-1}(\ell|[u](0)|+o(1)) \quad \text { as } j \uparrow \infty .
\end{aligned}
$$

Therefore, being $|[u](0)|<\ell^{-1}$, by the definition of $\widehat{F}$ we infer that

$$
\widehat{F}\left(u_{0}, 1 ; \delta Q_{e_{n}}\right) \leq \delta^{n-1}(1 \wedge \ell|[u](0)|),
$$

and estimate (7.5) follows at once.

Remark 7.2. The analysis in the general case of a diverging sequence $f^{(k)}$ is much more intricate because of the combination of several effects: the speed of divergence of the $f^{(k)}$ 's compared with the scaling $\varepsilon_{k}^{\frac{1}{2}}$ in the definition of $f_{k}^{(k)}$, and even more the behavior of each $f^{(k)}$ close to 1 . In this remark we limit ourselves to consider those families of functions $f^{(k)}$ satisfying item (ii) in Proposition 4.5, another instance shall be discussed in Remark 7.6 below.

Assume for example that $f(z)=\frac{\ell z}{1-z}$, and that $f^{(k)}$ is defined as in (7.2) above, with $a_{k}=\varepsilon_{k}^{-\frac{1}{2}}$, thus violating the last condition in (7.3). Then, one can show that the $\Gamma$-limit is given by the Mumford-Shah energy introduced in (2.1). Indeed, with this choice

$$
f_{k}^{(k)}(z)= \begin{cases}z & 0 \leq z \leq 1-\ell \varepsilon_{k}^{\frac{1}{2}} \\ \varepsilon_{k}^{\frac{1}{2}} \frac{\ell z}{1-z} & 1-\ell \varepsilon_{k}^{\frac{1}{2}} \leq z \leq\left(1+\ell \varepsilon_{k}^{\frac{1}{2}}\right)^{-1} \\ 1 & \left(1+\ell \varepsilon_{k}^{\frac{1}{2}}\right)^{-1} \leq z \leq 1,\end{cases}
$$

so that $f_{k}^{(k)}(z) \geq z$ for all $z \in[0,1]$, and actually $\left(f_{k}^{(k)}\right)$ converges uniformly to the identity on [0,1]. Therefore, $A T_{k}^{I d} \leq F_{k}^{(k)} \leq A T_{k}^{\psi}$, with $\psi(z)=\chi_{(0,1]}(z)$ (cp. with (2.2) for the definition of $A T_{k}^{\psi}$ ), and the assertion follows at once from Ambrosio and Tortorelli classical results (cp. [9], see also [30]).

A similar argument works also in the regime $a_{k} \varepsilon_{k}^{\frac{1}{2}} \uparrow \infty$, in which

$$
f_{k}^{(k)}(z)= \begin{cases}a_{k} \varepsilon_{k}^{\frac{1}{2}} z & 0 \leq z \leq a_{k}^{-1} \varepsilon_{k}^{-\frac{1}{2}} \\ 1 & a_{k}^{-1} \varepsilon_{k}^{-\frac{1}{2}} \leq z \leq 1,\end{cases}
$$

for $k$ sufficiently large, so that $f_{k}^{(k)}(z) \rightarrow \chi_{(0,1]}(z)$ for all $z \in[0,1]$, and again we get the Mumford-Shah energy in the $\Gamma$-limit arguing as above.

Finally, note that for $a_{k}$ as in (7.3), we have

$$
f_{k}^{(k)}(z)= \begin{cases}a_{k} \varepsilon_{k}^{\frac{1}{2}} z & 0 \leq z \leq 1-\ell a_{k}^{-1} \\ \varepsilon_{k}^{\frac{1}{2}} \frac{\ell z}{1-z} & 1-\ell a_{k}^{-1} \leq z \leq\left(1+\ell \varepsilon_{k}^{\frac{1}{2}}\right)^{-1} \\ 1 & \left(1+\ell \varepsilon_{k}^{\frac{1}{2}}\right)^{-1} \leq z \leq 1\end{cases}
$$

so that $f_{k}^{(k)}(z) \rightarrow \chi_{\{1\}}(z)$ in $[0,1]$. 


\subsection{A model with power-law growth at small openings}

In Theorem 7.4 below we approximate a model with sublinear surface density in the origin and quadratic growth for the volume term. To this end, let $p>1$ and consider a function $\psi_{p}$ satisfying condition (3.3) and

$$
\lim _{z \rightarrow 1^{-}}(1-z)^{p} \psi_{p}(z)=\gamma, \quad \gamma \in(0,+\infty) .
$$

Clearly, one can take $\psi_{p}(z):=\frac{\gamma z}{(1-z)^{p}}$ as prototype. The surface energy density $\vartheta_{p}:[0,+\infty) \rightarrow[0,+\infty)$ is defined as $g$ in (3.7) by

$$
\vartheta_{p}(s):=\inf _{(\alpha, \beta) \in \mathcal{U}_{s}} \int_{0}^{1}|1-\beta| \sqrt{\psi_{p}^{2}(\beta)\left|\alpha^{\prime}\right|^{2}+\left|\beta^{\prime}\right|^{2}} d t,
$$

where $\mathcal{U}_{S}$ has been introduced in (3.8). In this case the integral is finite only if $\beta<1$ almost everywhere on the set $\left\{\alpha^{\prime} \neq 0\right\}$. We next prove some properties of $\vartheta_{p}$ in analogy to Propositions 4.1, 4.3 and 4.5. In what follows, we keep the same notation introduced there. We also note that given any curve $(\alpha, \beta)$, the integral to be minimized in the definition of $\vartheta_{p}$ is invariant under reparametrizations of $(\alpha, \beta)$.

Proposition 7.3. Let $\psi_{p}$ satisfy (3.3) and (7.6), let $\vartheta_{p}:[0,+\infty) \rightarrow[0,+\infty)$ be the corresponding surface energy in (7.7). Then:

(i) $\vartheta_{p}(0)=0, \vartheta_{p}$ is nondecreasing, subadditive, and

$$
0 \leq \vartheta_{p}(s) \leq 1 \wedge c s^{\frac{2}{p+1}}, \quad \text { for all } s \geq 0,
$$

where $c=c\left(\psi_{p}\right)>0$. Moreover, $\vartheta_{p} \in C^{0, \frac{2}{p+1}}([0,+\infty))$ and

$$
\gamma^{\frac{2}{p+1}} \leq \lim _{s \downarrow 0} \frac{\vartheta_{p}(s)}{s^{\frac{2}{p+1}}} \leq c \gamma^{\frac{2}{p+1}}
$$

where $c=c(p)>0$;

(ii) $\vartheta_{p}=\hat{\vartheta}_{p}$, where

$$
\hat{\vartheta}_{p}(s):=\lim _{T \uparrow \infty} \inf _{(\alpha, \beta) \in \mathcal{U}_{s}(0, T)} \int_{0}^{T}\left(\psi_{p}^{2}(\beta)\left|\alpha^{\prime}\right|^{2}+\frac{(1-\beta)^{2}}{4}+\left|\beta^{\prime}\right|^{2}\right) d t ;
$$

(iii) the functions

$$
f^{(j)}(z):=\frac{j z}{1-z} \wedge \psi_{p}(z)
$$

satisfy (3.3) and (3.4). If $g_{j}$ denotes the corresponding surface energy in (3.7), then $g_{j} \leq g_{j+1}$ and

$$
\lim _{j \rightarrow \infty} g_{j}(s)=\vartheta_{p}(s) \quad \text { for all } s \geq 0 .
$$

Proof. We prove (i). The facts that $\vartheta_{p}(0)=0$ and that $\vartheta_{p}$ is nondecreasing follow easily from the definition. The subadditivity follows as in Proposition 4.1(i). Moreover, $0 \leq \vartheta_{p} \leq 1$ arguing as in (ii) of Proposition 4.1.

To show (7.8) and the upper bound in (7.9), let $s, \lambda>0$ and consider $\alpha:=0$ in $[0,1 / 3], \alpha:=s$ in $[2 / 3,1]$ and set $\alpha$ to be the linear interpolation of the values 0 and $s$ on $[1 / 3,2 / 3] ; \beta_{\lambda}:=1-(\lambda s)^{\frac{1}{p+1}}$ in $[1 / 3,2 / 3]$ and set $\beta_{\lambda}$ to be the linear interpolation of that value to 1 on $[0,1 / 3] \cup[2 / 3,1]$.

Then, clearly $\left(\alpha, \beta_{\lambda}\right) \in \mathcal{U}_{s}$ and a simple computation shows that

$$
\vartheta_{p}(s) \leq \int_{0}^{1}\left|1-\beta_{\lambda}\right| \sqrt{\psi_{p}^{2}\left(\beta_{\lambda}\right)\left|\alpha^{\prime}\right|^{2}+\left|\beta_{\lambda}^{\prime}\right|^{2}} d t=(\lambda s)^{\frac{1}{p+1}} \psi_{p}\left(1-(\lambda s)^{\frac{1}{p+1}}\right) s+(\lambda s)^{\frac{2}{p+1}} .
$$


By taking $\lambda=1$, since $(1-z)^{p} \psi_{p}(z) \leq c$ for some constant $c=c\left(\psi_{p}\right)>0$ and for all $z \in[0,1]$, we deduce that

$$
\vartheta_{p}(s) \leq(c+1) s^{\frac{2}{p+1}}
$$

from which inequality (7.8) follows as $0 \leq \vartheta_{p} \leq 1$.

The Hölder continuity of $\vartheta_{p}$ then follows easily from (7.8) and its subadditivity and monotonicity.

Further, by (7.12) we infer

$$
\limsup _{s \downarrow 0} \frac{\vartheta_{p}(s)}{s^{\frac{2}{p+1}}} \leq \gamma \lambda^{-\frac{p-1}{p+1}}+\lambda^{\frac{2}{p+1}},
$$

minimizing the latter inequality over $\lambda \in(0, \infty)$ yields the upper bound in (7.9).

We now prove the lower bound in (7.9). Let $s_{k} \rightarrow 0, s_{k}>0$, and up to subsequences let the liminf in (7.9) be a limit. Let $\alpha_{k}, \beta_{k}$ be competitors for $\vartheta_{p}\left(s_{k}\right)$ such that

$$
\int_{0}^{1}\left|1-\beta_{k}\right| \sqrt{\psi_{p}^{2}\left(\beta_{k}\right)\left|\alpha_{k}^{\prime}\right|^{2}+\left|\beta_{k}^{\prime}\right|^{2}} d t \leq \vartheta_{p}\left(s_{k}\right)+s_{k} .
$$

If, after taking a subsequence, there is a sequence $x_{j} \in[0,1]$ such that

$$
1-\beta_{j}\left(x_{j}\right) \geq\left(\gamma s_{j}\right)^{\frac{1}{p+1}} \text { for all } j,
$$

then

$$
\vartheta_{p}\left(s_{j}\right)+s_{j} \geq\left(1-\beta_{j}\left(x_{j}\right)\right)^{2} \geq\left(\gamma s_{j}\right)^{\frac{2}{p+1}} .
$$

Otherwise, for all $k$ large enough

$$
1-\beta_{k} \leq\left(\gamma s_{k}\right)^{\frac{1}{p+1}}
$$

must hold uniformly, so that $\beta_{k} \rightarrow 1$ uniformly and by (7.6) for any $\varepsilon>0$

$$
\left(1-\beta_{k}\right)^{p} \psi_{p}\left(\beta_{k}\right) \geq \gamma-\varepsilon \text { uniformly, for } k \text { large enough. }
$$

Therefore

$$
\vartheta_{p}\left(s_{k}\right)+s_{k} \geq \int_{0}^{1} \psi_{p}\left(\beta_{k}\right)\left(1-\beta_{k}\right)\left|\alpha_{k}^{\prime}\right| d t \geq \int_{0}^{1} \frac{\psi_{p}\left(\beta_{k}\right)\left(1-\beta_{k}\right)^{p}}{\left(1-\beta_{k}\right)^{p-1}}\left|\alpha_{k}^{\prime}\right| d t \geq \frac{\gamma-\varepsilon}{\gamma^{(p-1) /(p+1)}} s_{k}^{2 /(p+1)} .
$$

Since $\varepsilon$ was arbitrary this and (7.13) give the lower bound in (7.9).

Finally we prove that the limit in (7.9) exists. We fix a sequence $s_{j} \downarrow 0$ and choose $\alpha_{j}, \beta_{j} \in \mathcal{U}_{s_{j}}$ such that

$$
\int_{0}^{1}\left|1-\beta_{j}\right| \sqrt{\psi_{p}^{2}\left(\beta_{j}\right)\left|\alpha_{j}^{\prime}\right|^{2}+\left|\beta_{j}^{\prime}\right|^{2}} d t \leq \vartheta_{p}\left(s_{j}\right)+\frac{1}{j} s_{j}^{2 /(p+1)} .
$$

By the computation above we obtain $\beta_{j} \rightarrow 1$ uniformly. For $k \geq j$ we define $\alpha_{k}, \beta_{k} \in \mathcal{U}_{s_{k}}$ by

$$
\bar{\alpha}_{k}=\frac{s_{k}}{s_{j}} \alpha_{j} \text { and } \bar{\beta}_{k}=1-\left(\frac{s_{k}}{s_{j}}\right)^{1 /(p+1)}\left(1-\beta_{j}\right) .
$$

After a straightforward computation, using these test functions in the definition of $\vartheta_{p}\left(s_{k}\right)$ leads to

$$
\vartheta_{p}\left(s_{k}\right) \leq\left(\frac{s_{k}}{s_{j}}\right)^{2 /(p+1)}\left[\int_{0}^{1}\left|1-\beta_{j}\right| \sqrt{\psi_{p}^{2}\left(\beta_{j}\right)\left|\alpha_{j}^{\prime}\right|^{2}+\left|\beta_{j}^{\prime}\right|^{2}} d t\right] \sup \left\{\frac{\psi_{p}(z)(1-z)^{p}}{\psi_{p}\left(z^{\prime}\right)\left(1-z^{\prime}\right)^{p}}: \min \beta_{j} \leq z, z^{\prime}<1\right\} .
$$

Since $\beta_{j} \rightarrow 1$ uniformly as $j \rightarrow \infty$, and $\psi_{p}(z)(1-z)^{p}$ has a finite limit as $z \rightarrow 1$, the sup converges to 1 as $j \rightarrow \infty$. Therefore we obtain that for every $\varepsilon>0$ if $j$ is sufficiently large, then 


$$
\frac{\vartheta_{p}\left(s_{k}\right)}{s_{k}^{2 /(p+1)}} \leq(1+\varepsilon) \frac{\vartheta_{p}\left(s_{j}\right)}{s_{j}^{2 /(p+1)}}+\frac{1}{j} \quad \text { for all } k \geq j .
$$

This implies that the sequence converges. Since the decreasing sequence $s_{j}$ was arbitrary, the limit in (7.9) exists.

To establish (ii), we note first that by Cauchy's inequality $\vartheta_{p} \leq \hat{\vartheta}_{p}$. In order to prove the converse inequality, we first claim that $\alpha$ and $\beta$ in the infimum problem defining $\vartheta_{p}$ can be taken in $W^{1, \infty}((0,1))$. Let $\eta>0$ small and let $\alpha, \beta \in H^{1}((1 / 3,2 / 3))$ be competitors for $\vartheta_{p}(s)$ such that

$$
\int_{1 / 3}^{2 / 3}|1-\beta| \sqrt{\psi_{p}^{2}(\beta)\left|\alpha^{\prime}\right|^{2}+\left|\beta^{\prime}\right|^{2}} d t \leq \vartheta_{p}(s)+\eta .
$$

We define $\beta^{\eta}(t):=\beta(t) \wedge(1-\eta)$ in $[1 / 3,2 / 3]$. Since $(1-z)^{p} \psi_{p}(z)$ has a finite nonzero limit at 1 , there is a function $\omega$, with $\omega(\eta) \rightarrow 0$ as $\eta \rightarrow 0$, such that

$$
\left(1-z^{\prime}\right)^{p} \psi_{p}\left(z^{\prime}\right) \leq(1+\omega(\eta))(1-z)^{p} \psi_{p}(z) \text { for all } z, z^{\prime} \in[1-\eta, 1) .
$$

In particular, if $1-\eta<\beta(t)<1$, then

$$
\eta \psi_{p}(1-\eta) \leq \eta^{1-p}(1+\omega(\eta))(1-\beta(t))^{p} \psi_{p}(\beta(t)) \leq(1+\omega(\eta))(1-\beta(t)) \psi_{p}(\beta(t)) .
$$

We observe that $\beta^{\eta}=1-\eta$ and $\left(\beta^{\eta}\right)^{\prime}=0$ almost everywhere on the set $\left\{\beta \neq \beta^{\eta}\right\}$ and compute

$$
\begin{aligned}
\int_{\left\{\beta \neq \beta^{\eta}\right\}}\left(1-\beta^{\eta}\right) \sqrt{\psi_{p}^{2}\left(\beta^{\eta}\right)\left|\alpha^{\prime}\right|^{2}+\left|\left(\beta^{\eta}\right)^{\prime}\right|^{2}} d t & =\int_{\left\{\beta \neq \beta^{\eta}\right\}} \eta \psi_{p}(1-\eta)\left|\alpha^{\prime}\right| d t \\
& \leq(1+\omega(\eta)) \int_{\left\{\beta \neq \beta^{\eta}\right\}}(1-\beta) \psi_{p}(\beta)\left|\alpha^{\prime}\right| d t,
\end{aligned}
$$

so that by $(7.14)$ it follows

$$
\int_{1 / 3}^{2 / 3}\left|1-\beta^{\eta}\right| \sqrt{\psi_{p}^{2}\left(\beta^{\eta}\right)\left|\alpha^{\prime}\right|^{2}+\left|\left(\beta^{\eta}\right)^{\prime}\right|^{2}} d t \leq \vartheta_{p}(s)+\eta+\omega(\eta)+\eta \omega(\eta) .
$$

By density we are able to find two sequences $\alpha_{j}, \beta_{j}^{\eta} \in W^{1, \infty}((1 / 3,2 / 3))$ (actually in $C^{\infty}([1 / 3,2 / 3])$ ) such that $\alpha_{j}(1 / 3)=0, \alpha_{j}(2 / 3)=s, \beta_{j}^{\eta}(1 / 3)=\beta_{j}^{\eta}(2 / 3)=1-\eta, 0 \leq \beta \leq 1-\eta$, and converging respectively to $\alpha$ and $\beta^{\eta}$ in $H^{1}((1 / 3,2 / 3))$. Since the function $(1-z)^{p} \psi_{p}(z)$ is uniformly continuous in $[0,1-\eta]$ and since $\beta_{j}^{\eta} \rightarrow \beta^{\eta}$ also uniformly, we deduce that for $j$ large it holds

$$
\int_{1 / 3}^{2 / 3}\left|1-\beta_{j}^{\eta}\right| \sqrt{\psi_{p}^{2}\left(\beta_{j}^{\eta}\right)\left|\alpha_{j}^{\prime}\right|^{2}+\left|\left(\beta_{j}^{\eta}\right)^{\prime}\right|^{2}} d t \leq \vartheta_{p}(s)+2 \eta+\omega(\eta)+\eta \omega(\eta) .
$$

Finally we extend $\alpha_{j}$ and $\beta_{j}^{\eta}$ in $[0,1]$ defining $\alpha_{j}:=0$ in $[0,1 / 3], \alpha_{j}:=s$ in $[2 / 3,1]$, and $\beta_{j}^{\eta}$ as a linear interpolation of the values $1-\eta$ and 1 . Now $\alpha_{j}$ and $\beta_{j}^{\eta}$ are competitors for $\vartheta_{p}(s)$ and for $j$ large they satisfy

$$
\int_{0}^{1}\left|1-\beta_{j}^{\eta}\right| \sqrt{\psi_{p}^{2}\left(\beta_{j}^{\eta}\right)\left|\alpha_{j}^{\prime}\right|^{2}+\left|\left(\beta_{j}^{\eta}\right)^{\prime}\right|^{2}} d t \leq \vartheta_{p}(s)+2 \eta+\omega(\eta)+\eta \omega(\eta)+\eta^{2}
$$

and this concludes the proof of the claim.

Let us prove now that $\hat{\vartheta}_{p}(s) \leq \vartheta_{p}(s)$. We argue exactly as in Proposition 4.3 until estimate (4.8). In doing this we point out that $f, g$ and $\hat{g}$ have to be substituted by $\psi_{p}, \vartheta_{p}$ and $\hat{\vartheta}_{p}$, respectively.

By keeping the same notation introduced there, we repeat the computations in (7.15)-(7.17) and we conclude that 


$$
\hat{\vartheta}_{p}(s) \leq \sqrt{\eta}+3 \eta^{2}+(1+\omega(\eta)) \int_{0}^{1}(1-\beta) \sqrt{\psi_{p}^{2}(\beta)\left|\alpha^{\prime}\right|^{2}+\left|\beta^{\prime}\right|^{2}} d t .
$$

Since the last integral is less than $\vartheta_{p}(s)+\eta$ and $\eta$ can be made arbitrarily small the inequality $\hat{\vartheta}_{p} \leq \vartheta_{p}$ follows at once.

We now prove (iii). It is easy to check that $f^{(j)} \leq f^{(j+1)}$, and that $f^{(j)}(z) \rightarrow \psi_{p}(z)$ for all $z \in[0,1)$. Hence, the sequence $\left(g_{j}\right)$ is nondecreasing and $g_{j}(s) \leq \vartheta_{p}(s)$ for all $s \geq 0$. To prove (7.11), with fixed $s \in(0,+\infty)$, consider $\alpha_{j}, \beta_{j} \in W^{1, \infty}((1 / 3,2 / 3))$ such that $\alpha_{j}(1 / 3)=0, \alpha_{j}(2 / 3)=s, \beta_{j}(1 / 3)=\beta_{j}(2 / 3)=1$, and

$$
\int_{1 / 3}^{2 / 3}\left|1-\beta_{j}\right| \sqrt{\left(f^{(j)}\right)^{2}\left(\beta_{j}\right)\left|\alpha_{j}^{\prime}\right|^{2}+\left|\beta_{j}^{\prime}\right|^{2}} d t \leq g_{j}(s)+\frac{1}{j} .
$$

Let $\eta>0$ and assume first that $\inf \beta_{j_{k}} \leq \eta$ for a subsequence $j_{k} \uparrow \infty$. In this case, (7.18) yields

$$
g_{j_{k}}(s)+\frac{1}{j_{k}} \geq \int_{1 / 3}^{2 / 3}\left|1-\beta_{j} \| \beta_{j}^{\prime}\right| d t \geq(1-\eta)^{2},
$$

so that $\lim _{j} g_{j}(s)=\lim _{k} g_{j_{k}}(s) \geq 1 \geq \vartheta_{p}(s)$, which provides the conclusion.

Assume now that for all $j$ 's sufficiently large $\inf \beta_{j}>\eta$, and note that $f^{(j)}=\psi_{p}$ on $[\eta, 1-\eta]$ for all $j \geq j_{\eta}>0$. As we have already noticed in (7.16), if $\beta_{j}(t)>1-\eta$ we have

$$
\left(1-\beta_{j}(t)\right) \psi_{p}\left(\beta_{j}(t)\right) \geq \frac{1}{1+\omega(\eta)} \eta \psi_{p}(1-\eta),
$$

for some modulus of continuity $\omega$ of $(1-z)^{p} \psi_{p}(z)$ in 1 . Moreover, setting $c_{j}(\eta):=j \frac{(1-\eta)}{\eta \psi_{p}(1-\eta)} \wedge 1$, then

$$
j \beta_{j}(t)>j(1-\eta) \geq c_{j}(\eta) \eta \psi_{p}(1-\eta) .
$$

Note that $c_{j}(\eta) \rightarrow 1$ as $j \uparrow \infty$. Therefore, to compute the integral in (7.18) we separate the contributions on the sets $\left\{\beta_{j} \leq 1-\eta\right\},\left\{\beta_{j}>1-\eta\right\} \cap\left\{f^{j}=\psi_{p}\right\}$, and $\left\{\beta_{j}>1-\eta\right\} \cap\left\{f^{(j)}<\psi_{p}\right\}$ to get

$$
g_{j}(s)+\frac{1}{j} \geq\left(\frac{1}{1+\omega(\eta)} \wedge c_{j}(\eta)\right) \int_{1 / 3}^{2 / 3}\left|1-\beta_{j}^{\eta}\right| \sqrt{\psi_{p}^{2}\left(\beta_{j}^{\eta}\right)\left|\alpha_{j}^{\prime}\right|^{2}+\left|\left(\beta_{j}^{\eta}\right)^{\prime}\right|^{2}},
$$

where we have used (7.19), (7.20), and the fact that $f^{(j)}=\psi_{p}$ on $[\eta, 1-\eta]$. As before, we have employed the notation $\beta_{j}^{\eta}:=\beta_{j} \wedge(1-\eta)$. Extending now $\alpha_{j}$ and $\beta_{j}^{\eta}$ to the interval $(0,1)$ as in the previous step, the energy increases by $\eta^{2}$. Hence, for all $j$ sufficiently large it holds

$$
g_{j}(s)+\frac{1}{j} \geq\left(\frac{1}{1+\omega(\eta)} \wedge c_{j}(\eta)\right)\left(\vartheta_{p}(s)-\eta^{2}\right),
$$

and letting first $j \uparrow \infty$ and then $\eta \downarrow 0$ we conclude (7.11).

The functionals $F_{k}^{(k)}$ corresponding to the sequence $\left(f^{(j)}\right)$ in (7.10) of Proposition 7.3 provide an approximation of $\Phi_{p}: L^{1}(\Omega) \rightarrow[0,+\infty]$ defined by

$$
\Phi_{p}(u):= \begin{cases}\int_{\Omega}|\nabla u|^{2} d x+\int_{J_{u}} \vartheta_{p}(|[u]|) d \mathcal{H}^{n-1} & \text { if } u \in \operatorname{GSBV}(\Omega), \\ +\infty & \text { otherwise, }\end{cases}
$$

with $\vartheta_{p}$ defined in formula (7.7). 
Theorem 7.4. Suppose that $\left(f^{(j)}\right)$ is as in (7.10) above.

Then, the functionals $F_{k}^{(k)}$ defined in (7.1) $\Gamma$-converge in $L^{1}(\Omega) \times L^{1}(\Omega)$ to $\widetilde{\Phi}_{p}$, where

$$
\widetilde{\Phi}_{p}(u, v):= \begin{cases}\Phi_{p}(u) & \text { if } v=1 \mathcal{L}^{n} \text {-a.e. in } \Omega, \\ +\infty & \text { otherwise. }\end{cases}
$$

Proof. By monotonicity of the sequence $\left(f_{k}^{(j)}\right)$ we have that $F_{k}^{(k)} \geq F_{k}^{(j)}$ for $k \geq j$, so that by Theorem 3.1 if $\Gamma$ - $\liminf _{k} F_{k}^{(k)}(u, v)<+\infty$ then $u \in G B V(\Omega), v=1 \mathcal{L}^{n}$-a.e. on $\Omega$ and for all $j \in \mathbb{N}$

$$
\Gamma-\liminf _{k} F_{k}^{(k)}(u, 1) \geq \Gamma-\lim _{k} F_{k}^{(j)}(u, 1)=\int_{\Omega} h_{j}(|\nabla u|) d x+\int_{J_{u}} g_{j}(|[u]|) d \mathcal{H}^{n-1}+j\left|D^{c} u\right|(\Omega),
$$

where $h_{j}$ and $g_{j}$ are defined, respectively, by (3.6) and (3.7) with $f^{(j)}$ in place of $f$. By letting $j \uparrow \infty$, we get that

$$
h_{j}(s) \uparrow s^{2}, \quad \text { and } \quad g_{j}(s) \uparrow \vartheta_{p}(s) \text { for all } s \geq 0 .
$$

Indeed, the former convergence follows from the explicit formula $h_{j}(s)=s^{2}$ for $s \in[0, j / 2]$ and $h_{j}(s)=j s-j^{2} / 4$ for $s \in[j / 2,+\infty)$, while the latter in view of (iii) in Proposition 7.3. Therefore, by Beppo-Levi's theorem we conclude that $u \in \operatorname{GSBV}(\Omega)$ with

$$
\Gamma-\liminf _{k} F_{k}^{(k)}(u, 1) \geq \widetilde{\Phi}_{p}(u, 1) .
$$

To prove the upper bound inequality we note that Lemmas 6.2 and 6.3 still hold true in this setting as there we have only used that each function $f_{k}=1 \wedge \varepsilon_{k}^{\frac{1}{2}} f$ in (3.2) is nondecreasing and bounded by 1 from above, properties enjoyed by $f_{k}^{(k)}$ as well (cp. also Theorem 7.1).

Hence, we may argue again as in Proposition 6.4 and reduce ourselves to prove the estimate

$$
\underset{\delta \downarrow 0}{\limsup } \frac{1}{\delta^{n-1}} \widehat{F}\left(u_{x}, 1 ; x+\delta Q_{v_{u}(x)}\right) \leq \vartheta_{p}(|[u](x)|),
$$

for $u \in S B V^{2}(\Omega)$ and for $\mathcal{H}^{n-1}$-a.e. $x \in J_{u}$, where $\widehat{F}$ is the $\bar{\Gamma}$-limit of a properly chosen subsequence $\left(F_{k_{j}}^{\left(k_{j}\right)}\right)$ of $\left(F_{k}^{(k)}\right)$. Given (7.21), we deduce the upper bound estimate as follows: we employ first [13, Propositions 3.3-3.5] to get the estimate $\widehat{F}(\cdot, 1) \leq \widetilde{\Phi}_{p}(\cdot, 1)$ on the full $S B V$ space, by relaxing the functional $\Phi_{\infty}: B V(\Omega) \rightarrow[0,+\infty]$

$$
\Phi_{\infty}(u):= \begin{cases}\int_{\Omega}|\nabla u|^{2} d x+\int_{J_{u}} \vartheta_{p}(|[u](x)|) d \mathcal{H}^{n-1} & \text { if } u \in S B V^{2}(\Omega) \\ +\infty & \text { otherwise on } B V(\Omega),\end{cases}
$$

w.r.t. the weak- $*-B V$ topology on $B V(\Omega)$. This implies $\widehat{F}(\cdot, 1) \leq \Phi_{p}$ on $B V(\Omega)$. We get the required estimate on the whole $G S B V \cap L^{1}(\Omega)$ by the usual truncation argument. We then argue as in Proposition 6.4 to show that the whole family $\left(F_{k}^{(k)}\right) \Gamma$-converges to $\widetilde{\Phi}_{p}$.

The proof of (7.21) is identical to the proof of (6.18) in Proposition 6.4 and therefore not repeated.

\subsection{Griffith's brittle fracture}

Finally, we show how to approximate the Mumford-Shah functional by means of any sequence $\left(f^{(j)}\right)$ satisfying item (iii) in Proposition 4.5. Thus, we recover the original approximation scheme of Ambrosio and Tortorelli $[8,9]$ (see also [30]).

Theorem 7.5. Suppose that $\left(f^{(j)}\right)$ satisfies $f^{(j)} \leq f^{(j+1)}, \ell_{j} \uparrow \infty$ and $f^{(j)}(z) \uparrow \infty$ pointwise in $(0,1)$. Then, the functionals $F_{k}^{(k)} \Gamma$-converge in $L^{1}(\Omega) \times L^{1}(\Omega)$ to the functional $\widetilde{M S}$ defined as follows

$$
\widetilde{M S}(u, v):= \begin{cases}M S(u) & \text { if } v=1 \mathcal{L}^{n} \text {-a.e. in } \Omega, \\ +\infty & \text { otherwise. }\end{cases}
$$


Proof. As in the proof of Theorem 7.1 we first note that $F_{k}^{(j)} \leq F_{k}^{(k)}$ for $j \leq k$. Thus, by Theorem 3.1 we deduce

$$
\Gamma-\liminf _{k} F_{k}^{(k)}(u, v) \geq F^{(j)}(u, v),
$$

where $F^{(j)}$ is defined as $F$ in (3.9) with $f$ substituted by $f^{(j)}$ in formulas (3.6) defining $h_{j}$, and (3.7) defining $g_{j}$. In particular, the corresponding volume density is given by

$$
h_{j}(s)= \begin{cases}s^{2} & s \leq \frac{\ell_{j}}{2} \\ \ell_{j} s-\frac{\ell_{j}^{2}}{4} & s \geq \frac{\ell_{j}}{2},\end{cases}
$$

where $\ell_{j}$ is the value of the limit in (3.4) and it satisfies $\ell_{j} \uparrow \infty$. Thus $h_{j}(s) \leq s^{2}$ and $\lim _{j} h_{j}(s)=s^{2}$ for all $s \in[0,+\infty)$. Moreover, the surface energy densities $g_{j}$ are dominated by the constant 1 , and by item (iii) in Proposition 4.5 we have $\lim _{j} g_{j}(s)=\chi_{(0,+\infty)}(s)$ for all $s \in[0,+\infty)$. In conclusion, if $\Gamma$ - $\liminf _{k} F_{k}^{(k)}(u, v)<+\infty$, by letting $j \uparrow \infty$ in (7.22) we infer that $v=1 \mathcal{L}^{n}$-a.e. in $\Omega, u \in G S B V(\Omega)$ and by Beppo-Levi's theorem we get

$$
\Gamma-\liminf _{k} F_{k}^{(k)}(u, v) \geq \widetilde{M S}(u) .
$$

Finally, we establish the limsup inequality. Set $\psi:=\chi_{(0,1]}$, we observe once more that $F_{k}^{(k)} \leq A T_{k}^{\psi}$ for every $k$, where $A T_{k}^{\psi}$ has been defined in (2.2). Therefore the conclusion follows by the Ambrosio and Tortorelli result [9] (see also [30]).

Remark 7.6. In Remark 7.2 we have shown that both the divergence of the $f_{k}$ 's and the scaling with $\varepsilon_{k}^{\frac{1}{2}}$ in the definition of $f_{k}^{(k)}$ are influencing the asymptotic behavior of the related sequence $\left(F_{k}^{(k)}\right)$. Here, we show that also the sequence of values of the limits in 1 of the functions $(1-z) f^{(k)}(z)$, i.e. $\ell_{k}$, is playing a role. In particular, we highlight that the pointwise limit of $\left(f_{k}^{(k)}\right)$ alone does not determine the asymptotics of $\left(F_{k}^{(k)}\right)$.

Indeed, if $f^{(k)}(z):=a_{k} \frac{z}{1-z}$, where $a_{k} \uparrow \infty$, then

$$
f_{k}^{(k)}(z)= \begin{cases}a_{k} \varepsilon_{k}^{\frac{1}{2}} \frac{z}{1-z} & 0 \leq z \leq\left(1+a_{k} \varepsilon_{k}^{\frac{1}{2}}\right)^{-1} \\ 1 & \left(1+a_{k} \varepsilon_{k}^{\frac{1}{2}}\right)^{-1} \leq z \leq 1,\end{cases}
$$

and by letting $k \uparrow \infty$ we infer that

$$
f_{k}^{(k)}(z) \rightarrow \begin{cases}\chi_{\{1\}}(z) & \text { if } a_{k} \varepsilon_{k}^{\frac{1}{2}} \downarrow 0 \\ \gamma \frac{z}{1-z} \wedge 1 & \text { if } a_{k} \varepsilon_{k}^{\frac{1}{2}} \rightarrow \gamma \in(0,+\infty) \\ \chi_{(0,1]}(z) & \text { if } a_{k} \varepsilon_{k}^{\frac{1}{2}} \uparrow \infty .\end{cases}
$$

Hence, by taking also into account the examples in Remark 7.2, we have built two sequences of functions both converging to $\chi_{\{1\}}$ but giving rise in the $\Gamma$-limit on one hand to Dugdale's cohesive energy and on the other hand to Griffith's type energy. In the first example $\ell_{k}$ are constant, in the second $\ell_{k}$ diverge.

\section{Conflict of interest statement}

The authors declare there is no conflict of interest.

\section{Acknowledgements}

Part of this work was conceived when M. Focardi was visiting the University of Bonn in winter 2014. He would like to thank the Institute for Applied Mathematics for the hospitality and the stimulating scientific atmosphere provided during his stay. 
M. Focardi and F. Iurlano are grateful to Gianni Dal Maso for stimulating discussions and for many insightful remarks. They are members of the Gruppo Nazionale per l'Analisi Matematica, la Probabilità e le loro Applicazioni (GNAMPA) of the Istituto Nazionale di Alta Matematica (INdAM).

F. Iurlano was funded under a postdoctoral fellowship by the Hausdorff Center for Mathematics. She is a member of the ERC Project No. 290888 "Quasistatic and Dynamic Evolution Problems in Plasticity and Fracture".

\section{References}

[1] G. Alberti, G. Bouchitté, P. Seppecher, Phase transition with the line-tension effect, Arch. Ration. Mech. Anal. 144 (1998) 1-46.

[2] R. Alicandro, A. Braides, J. Shah, Free-discontinuity problems via functionals involving the $L^{1}$-norm of the gradient and their approximations, Interfaces Free Bound. 1 (1999) 17-37.

[3] R. Alicandro, M. Focardi, Variational approximation of free-discontinuity energies with linear growth, Commun. Contemp. Math. 4 (2002) $685-723$.

[4] L. Ambrosio, A compactness theorem for a new class of functions of bounded variation, Boll. Unione Mat. Ital., B (7) 3 (1989) $857-881$

[5] L. Ambrosio, L. Faina, R. March, Variational approximation of a second order free discontinuity problem in computer vision, SIAM J. Math. Anal. 32 (2001) 1171-1197.

[6] L. Ambrosio, N. Fusco, D. Pallara, Functions of Bounded Variation and Free Discontinuity Problems, Oxf. Math. Monogr., The Clarendon Press, Oxford University Press, New York, 2000.

[7] L. Ambrosio, A. Lemenant, G. Royer-Carfagni, A variational model for plastic slip and its regularization via $\Gamma$-convergence, J. Elast. 110 (2013) 201-235.

[8] L. Ambrosio, V.M. Tortorelli, Approximation of functionals depending on jumps by elliptic functionals via $\Gamma$-convergence, Commun. Pure Appl. Math. 43 (1990) 999-1036.

[9] L. Ambrosio, V.M. Tortorelli, On the approximation of free discontinuity problems, Boll. Unione Mat. Ital., B (7) 6 (1992) $105-123$.

[10] L. Bar, N. Sochen, N. Kiryati, Image deblurring in the presence of impulsive noise, Int. J. Comput. Vis. 70 (2006) $279-298$.

[11] G.I. Barenblatt, The mathematical theory of equilibrium cracks in brittle fracture, in: Adv. Appl. Mech., vol. 7, Academic Press, New York, 1962, pp. 55-129.

[12] G. Bellettini, A. Coscia, Discrete approximation of a free discontinuity problem, Numer. Funct. Anal. Optim. 15 (1994) $201-224$.

[13] G. Bouchitté, A. Braides, G. Buttazzo, Relaxation results for some free discontinuity problems, J. Reine Angew. Math. 458 (1995) 1-18.

[14] G. Bouchitté, I. Fonseca, G. Leoni, L. Mascarenhas, A global method for relaxation in $W^{1, p}$ and in $\mathrm{SBV}_{p}$, Arch. Ration. Mech. Anal. 165 (2002) 187-242.

[15] B. Bourdin, Numerical implementation of the variational formulation for quasi-static brittle fracture, Interfaces Free Bound. 9 (2007) 411-430.

[16] B. Bourdin, G.A. Francfort, J.-J. Marigo, Numerical experiments in revisited brittle fracture, J. Mech. Phys. Solids 48 (2000) 797-826.

[17] B. Bourdin, G.A. Francfort, J.-J. Marigo, The variational approach to fracture, J. Elast. 91 (2008) 5-148.

[18] A. Braides, Г-Convergence for Beginners, Oxford Lecture Ser. Math. Appl., vol. 22, Oxford University Press, Oxford, 2002

[19] A. Braides, G. Dal Maso, A. Garroni, Variational formulation of softening phenomena in fracture mechanics: the one-dimensional case, Arch. Ration. Mech. Anal. 146 (1999) 23-58.

[20] A. Braides, M.S. Gelli, From discrete systems to continuous variational problems: an introduction, in: Topics on Concentration Phenomena and Problems with Multiple Scales, in: Lect. Notes Unione Mat. Ital., vol. 2, Springer, Berlin, 2006, pp. 3-77.

[21] S. Burke, C. Ortner, E. Süli, An adaptive finite element approximation of a variational model of brittle fracture, SIAM J. Numer. Anal. 48 (2010) 980-1012.

[22] S. Burke, C. Ortner, E. Süli, An adaptive finite element approximation of a generalized Ambrosio-Tortorelli functional, Math. Models Methods Appl. Sci. 23 (2013) 1663-1697.

[23] A. Chambolle, An approximation result for special functions with bounded deformation, J. Math. Pures Appl. (9) 83 (2004) 929-954.

[24] A. Chambolle, Addendum to: "An approximation result for special functions with bounded deformation" [J. Math. Pures Appl. (9) 83 (7) (2004) 929-954], J. Math. Pures Appl. (9) 84 (2005) 137-145.

[25] G. Dal Maso, An Introduction to Г-Convergence, Prog. Nonlinear Differ. Equ. Appl., vol. 8, Birkhäuser Boston Inc., Boston, MA, 1993.

[26] G. Dal Maso, F. Iurlano, Fracture models as $\Gamma$-limits of damage models, Commun. Pure Appl. Anal. 12 (2013) 1657-1686.

[27] G. del Piero, L. Truskinovsky, A one-dimensional model for localized and distributed failure, J. Phys. IV 8 (1998) 95-102.

[28] G. del Piero, L. Truskinovsky, Macro- and micro-cracking in one-dimensional elasticity, Int. J. Solids Struct. 38 (2001) $1135-1148$.

[29] D. Dugdale, Yielding of steel sheets containing slits, J. Mech. Phys. Solids 8 (1960) 100-108.

[30] M. Focardi, On the variational approximation of free-discontinuity problems in the vectorial case, Math. Models Methods Appl. Sci. 11 (2001) $663-684$.

[31] M. Focardi, Variational approximation of vectorial free discontinuity problems: the discrete and continuous case, PhD thesis, Scuola Normale Superiore, Pisa, 2002.

[32] M. Focardi, F. Iurlano, Asymptotic analysis of Ambrosio-Tortorelli energies in linearized elasticity, SIAM J. Math. Anal. 46 (2014) $2936-2955$

[33] L. Fokoua, S. Conti, M. Ortiz, Optimal scaling laws for ductile fracture derived from strain-gradient microplasticity, J. Mech. Phys. Solids 62 (2014) 295-311.

[34] G.A. Francfort, J.-J. Marigo, Stable damage evolution in a brittle continuous medium, Eur. J. Mech. A, Solids 12 (1993) 149-189.

[35] G.A. Francfort, J.-J. Marigo, Revisiting brittle fracture as an energy minimization problem, J. Mech. Phys. Solids 46 (1998) $1319-1342$.

[36] N. Fusco, An overview of the Mumford-Shah problem, Milan J. Math. 71 (2003) 95-119. 
[37] D. Henao, C. Mora-Corral, X. Xu, Г-convergence approximation of fracture and cavitation in nonlinear elasticity, Arch. Ration. Mech. Anal. (2014) 1-67, http://dx.doi.org/10.1007/s00205-014-0820-3.

[38] F. Iurlano, Fracture and plastic models as $\Gamma$-limits of damage models under different regimes, Adv. Calc. Var. 6 (2013) $165-189$.

[39] F. Iurlano, A density result for GSBD and its application to the approximation of brittle fracture energies, Calc. Var. Partial Differ. Equ. 51 (2014) 315-342.

[40] K. Pham, J.-J. Marigo, Approche variationnelle de l'endommagement: I. Les concepts fondamentaux, C. R., Méc. 338 (2010) $191-198$.

[41] K. Pham, J.-J. Marigo, Approche variationnelle de l'endommagement: II. Les modèles à gradient, C. R., Méc. 338 (2010) $199-206$.

[42] J. Shah, Curve evolution and segmentation functionals: application to color images, in: Proceedings IEEE ICIP, 1996. 